Homology, Homotopy and Applications, vol.5(1), 2003, pp.297-324

\title{
REPRESENTATION TYPES AND 2-PRIMARY HOMOTOPY GROUPS OF CERTAIN COMPACT LIE GROUPS
}

\author{
DONALD M. DAVIS \\ (communicated by Gunnar Carlsson)
}

\begin{abstract}
Bousfield has shown how the 2-primary $v_{1}$-periodic homotopy groups of certain compact Lie groups can be obtained from their representation ring with its decomposition into types and its exterior power operations. He has formulated a Technical Condition which must be satisfied in order that he can prove that his description is valid.

We prove that a simply-connected compact simple Lie group satisfies his Technical Condition if and only if it is not $E_{6}$ or $\operatorname{Spin}(4 k+2)$ with $k$ not a 2 -power. We then use his description to give an explicit determination of the 2-primary $v_{1}$-periodic homotopy groups of $E_{7}$ and $E_{8}$. This completes a program, suggested to the author by Mimura in 1989, of computing the $v_{1}$-periodic homotopy groups of all compact simple Lie groups at all primes.
\end{abstract}

\section{Introduction}

The $p$-primary $v_{1}$-periodic homotopy groups of a topological space $X$, denoted $v_{1}^{-1} \pi_{*}(X ; p)$, are a localization of the portion of the actual homotopy groups detected by $K$-theory. Each $v_{1}$-periodic homotopy group of $X$ is a direct summand of some actual homotopy group of $X$.

In 1989, Mimura suggested to the author that the computation of $v_{1}^{-1} \pi_{*}(X ; p)$ for all compact simple Lie groups $X$ and all primes $p$ would be an interesting project. In a series of papers over the subsequent 13-year period, the author, often in collaboration with Bendersky, had performed this computation in all cases except

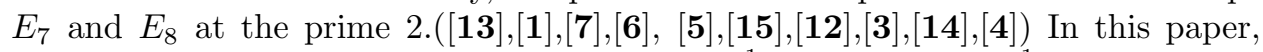
we use recent work of Bousfield to compute $v_{1}^{-1} \pi_{*}\left(E_{7} ; 2\right)$ and $v_{1}^{-1} \pi_{*}\left(E_{8} ; 2\right)$, thus completing the project suggested by Mimura.

The one impreciseness in these results is some extension questions involving $\mathbf{Z}_{2}$ 's. We write $\mathbf{Z}_{2}$ interchangeably with $\mathbf{Z} / 2$. We denote by $A \# B$ an abelian group $G$

The author would like to thank Pete Bousfield for his suggestions on this work and for allowing him to use his unpublished results.

Received April 21, 2003, revised August 21, 2003; published on September 4, 2003.

2000 Mathematics Subject Classification: 55Q52,55T15,57T20

Key words and phrases: homotopy groups, exceptional Lie groups, representation theory

(C) 2003, Donald M. Davis. Permission to copy for private use granted. 
such that there is a short exact sequence of abelian groups

$$
0 \rightarrow A \rightarrow G \rightarrow B \rightarrow 0 \text {. }
$$

Here and throughout the remainder of the paper $v_{1}^{-1} \pi_{*}(X)$ means $v_{1}^{-1} \pi_{*}(X ; 2)$. Also, $\nu(-)$ denotes the exponent of 2 in an integer.

Theorem 1.1. For all integers $k$, there are isomorphisms

$$
v_{1}^{-1} \pi_{8 k+d}\left(E_{7}\right) \approx \begin{cases}\mathbf{Z}_{2} & d=3 \\ \mathbf{Z}_{2} \# \mathbf{Z}_{2} & d=4 \\ \mathbf{Z}_{2} \oplus \mathbf{Z} / 2^{4} \oplus \mathbf{Z} / 2^{g(4 k+3)-2} & d=5 \\ \mathbf{Z} / 4 \oplus \mathbf{Z} / 2^{g(4 k+3)} \oplus \mathbf{Z}_{2} \oplus \mathbf{Z}_{2} & d=6 \\ \left(\mathbf{Z}_{2} \oplus \mathbf{Z}_{2}\right) \#\left(\mathbf{Z}_{2} \oplus \mathbf{Z}_{2} \oplus \mathbf{Z}_{2}\right) & d=7 \\ \left(\mathbf{Z}_{2} \oplus \mathbf{Z}_{2} \oplus \mathbf{Z}_{2}\right) \# \mathbf{Z}_{2} & d=8 \\ \mathbf{Z}_{2} \oplus \mathbf{Z} / 2^{4} \oplus \mathbf{Z} / 2^{g(4 k+5)-2} & d=9 \\ \mathbf{Z} / 4 \oplus \mathbf{Z} / 2^{g(4 k+5)} & d=10,\end{cases}
$$

where

$$
g(m)=\left\{\begin{array}{lll}
\min \left(17, \nu\left(m-11-2^{6}\right)+9\right) & m \equiv 3 & \bmod 4 \\
\min \left(18, \nu\left(m-13-2^{7}\right)+9\right) & m \equiv 5 & \bmod 8 \\
\min \left(23, \nu\left(m-17-7 \cdot 2^{11}\right)+9\right) & m \equiv 1 & \bmod 8
\end{array}\right.
$$

Theorem 1.2. For all integers $k$, there are isomorphisms

$$
v_{1}^{-1} \pi_{8 k+d}\left(E_{8}\right) \approx \begin{cases}\mathbf{Z} / 2^{e(4 k-1)-1} \oplus \mathbf{Z}_{2} & d=-3 \\ \mathbf{Z} / 2^{e(4 k-1)} \oplus \mathbf{Z}_{2} \oplus \mathbf{Z}_{2} & d=-2 \\ \left(\mathbf{Z}_{2} \oplus \mathbf{Z}_{2} \oplus \mathbf{Z}_{2}\right) \#\left(\mathbf{Z}_{2} \oplus \mathbf{Z}_{2}\right) & d=-1 \\ \left(\mathbf{Z}_{2} \# \mathbf{Z}_{2}\right) \oplus \mathbf{Z}_{2} \oplus \mathbf{Z}_{2} & d=0 \\ \mathbf{Z} / 2^{e(4 k+1)-1} \oplus \mathbf{Z}_{2} \oplus \mathbf{Z}_{2} & d=1 \\ \mathbf{Z} / 2^{e(4 k+1)} & d=2 \\ 0 & d=3,4,\end{cases}
$$

where

$$
e(m)=\left\{\begin{array}{lll}
\min \left(25, \nu\left(m-17-2^{8}-2^{11}-2^{12}\right)+12\right) & m \equiv 1 & \bmod 8 \\
\min \left(28, \nu\left(m-19-2^{11}-2^{14}-2^{15}\right)+12\right) & m \equiv 3 & \bmod 8 \\
\min \left(39, \nu\left(m-29-2^{20}-2^{22}-2^{23}-2^{25}\right)+12\right) & m \equiv 5 & \bmod 8 \\
\min \left(31, \nu\left(m-23-2^{17}\right)+12\right) & m \equiv 7 & \bmod 8
\end{array}\right.
$$

Note that the numbers $m_{0}=17,19,29$, and 23 which occur in $\nu\left(m-m_{0}-2^{L}\right)$ in the formula for $e(m)$ are the largest exponents of $E_{8}$, and similarly for $E_{7}$ with $m_{0}=11,13$, and 17 . The exponents of a compact Lie group $G$ are those integers $m_{i}$ such that $H^{*}(G ; \mathbf{Q})$ is an exterior algebra on classes of grading $2 m_{i}+1 .([\mathbf{1 0}$, pp.15-16].)

In 2.2, we state a slight reformulation of a conjecture of Bousfield that would yield, for all simply-connected compact Lie groups $G$, the groups $v_{1}^{-1} \pi_{*}(G ; 2)$ in 
terms of the representation ring $R(G)$ together with its decomposition into real, complex, and quaternionic types, and its second and third exterior power operations. Bousfield has proved (see Theorem 2.5) that his conjecture is valid for those $G$ for which $R(G)$ satisfies a Technical Condition, which we state in 2.4. Our second main result determines which of the simply-connected compact simple Lie groups satisfy this Technical Condition.

Theorem 1.3. A simply-connected compact simple Lie group satisfies the Technical Condition 2.4 if and only if it is not $E_{6}$ or $\operatorname{Spin}(4 k+2)$ with $k$ not a 2-power.

In particular, Conjecture 2.2 is valid for $E_{7}$ and $E_{8}$. It is by computing the groups and homomorphisms of 2.2 that Theorems 1.1 and 1.2 are proved.

The author has computed, for all compact simple Lie groups $G$, the result for $v_{1}^{-1} \pi_{*}(G ; 2)$ which would be implied by Bousfield's conjecture 2.2 and obtained remarkable agreement with the results he has obtained previously by other methods. This may be viewed both as lending credence to Conjecture 2.2 and as a check on the earlier work of the author and coworkers.

\section{Bousfield's Conjecture and Theorem}

In this section, we state a slight reformulation of Bousfield's conjecture regarding 2-primary $v_{1}$-periodic homotopy groups, and his Technical Condition, under which he can prove his conjecture valid.

The first step of Bousfield's program is a real analogue of $[\mathbf{8}, 8.1,8.5]$. In a November 2002 e-mail, Bousfield wrote that the following result can be proved by utilizing $[\mathbf{9}, 7.8,9.4,9.5]$ to adapt the argument of $[\mathbf{8}]$.

Theorem 2.1. (Bousfield) Let $G$ be a simply-connected compact Lie group. There is a $K / 2_{*}$-local spectrum $\Phi G$ such that there is an exact sequence

$$
\rightarrow v_{1}^{-1} \pi_{i+2}(G)^{\#} \rightarrow K O^{i}\left(\Phi G ; \mathbf{Z}_{2}^{\wedge}\right) \stackrel{\psi^{3}-9}{\longrightarrow} K O^{i}\left(\Phi G ; \mathbf{Z}_{2}^{\wedge}\right) \rightarrow v_{1}^{-1} \pi_{i+3}(G)^{\#} \rightarrow,
$$

where (-)\# denotes Pontrjagin duality.

Bousfield's conjecture expresses $K O^{*}\left(\Phi G ; \mathbf{Z}_{2}^{\wedge}\right)$ in terms of the representation theory of $G$. For the simply-connected compact Lie group $G$, let $R(G)$ be its (complex) representation ring, $I \subset R(G)$ the augmentation ideal, and $Q=Q(G)=I / I^{2}$ the group of indecomposables in $I$. Let $R_{\mathbf{R}}(G)$ (resp. $R_{\mathbf{H}}(G)$ ) denote the real (resp. quaternionic) representation rings. We identify these with their image in $R(G)$ under the extension homomorphisms, which are injective. Let $Q_{\mathbf{R}} \subset Q\left(\right.$ resp. $\left.Q_{\mathbf{H}} \subset Q\right)$ denote the image in $Q$ of the augmentation ideal of $R_{\mathbf{R}}(G)$ (resp. $R_{\mathbf{H}}(G)$ ). Let $\lambda^{k}$ denote the exterior power operations on $R(G)$.

The following conjecture uses all the above notation. We omit writing $\mathbf{Z}_{2}^{\wedge}$ as coefficient of $K O^{*}(\Phi G)$, and a $\mathbf{Z}_{2}^{\wedge} \otimes$ which should accompany all the $Q$-groups. Although essential to the underlying theory, these 2 -adic coefficients do not affect the subsequent calculations. 
Conjecture 2.2. (Bousfield) If $G$ is a simply-connected compact Lie group, there is an exact sequence of abelian groups

$$
\begin{aligned}
0 & \rightarrow K O^{0}(\Phi G) \rightarrow Q /\left(Q_{\mathbf{R}}+Q_{\mathbf{H}}\right) \stackrel{\lambda^{2}}{\longrightarrow} Q / Q_{\mathbf{R}} \rightarrow K O^{1}(\Phi G) \rightarrow 0 \\
& \rightarrow Q_{\mathbf{H}} /\left(Q_{\mathbf{R}} \cap Q_{\mathbf{H}}\right) \rightarrow K O^{2}(\Phi G) \rightarrow Q_{\mathbf{R}} \cap Q_{\mathbf{H}} \stackrel{\lambda^{2}}{\longrightarrow} Q_{\mathbf{H}} \rightarrow K O^{3}(\Phi G) \rightarrow 0 \\
& \rightarrow K O^{4}(\Phi G) \rightarrow Q /\left(Q_{\mathbf{R}} \cap Q_{\mathbf{H}}\right) \stackrel{\lambda^{2}}{\longrightarrow} Q / Q_{\mathbf{H}} \rightarrow K O^{5}(\Phi G) \\
& \rightarrow\left(Q_{\mathbf{R}}+Q_{\mathbf{H}}\right) /\left(Q_{\mathbf{R}} \cap Q_{\mathbf{H}}\right) \stackrel{\lambda^{2}}{\longrightarrow} Q_{\mathbf{R}} /\left(Q_{\mathbf{R}} \cap Q_{\mathbf{H}}\right) \rightarrow K O^{6}(\Phi G) \\
& \rightarrow Q_{\mathbf{R}}+Q_{\mathbf{H}} \stackrel{\lambda^{2}}{\longrightarrow} Q_{\mathbf{R}} \rightarrow K O^{7}(\Phi G) \rightarrow 0 .
\end{aligned}
$$

For any integer $i$, the Adams operation $\psi^{3}$ in $K O^{2 i}(\Phi G)$ and $K O^{2 i+1}(\Phi G)$ corresponds to $3^{-i} \lambda^{3}$ in $Q$ under the morphisms of the exact sequence, which is expanded to all integers by Bott periodicity $K O^{j}(-) \approx K O^{j+8}(-)$.

Note that applying period- 8 Bott periodicity to the exact sequence of 2.2 does not change the $\lambda^{2}$ in $Q$ which is being used to yield the $K O^{*}(-)$-groups. We will show in Sections 4 and 5 how to compute the exact sequences of Theorem 2.1 and Conjecture 2.2 to obtain $v_{1}^{-1} \pi_{*}\left(E_{8}\right)$ and $v_{1}^{-1} \pi_{*}\left(E_{7}\right)$.

Bousfield has proved this conjecture for those $G$ which satisfy a Technical Condition, which we now state. We begin by recalling some standard material regarding representation types, and establishing notation. All of the material in this result, as well as additional background for 2.4, may be found in [11, II.6,VI.4].

Theorem 2.3. Let $G$ be a simply-connected compact Lie group, and let $t$ denote conjugation on $R(G)$. Each irreducible representation is of one of three typesreal, quaternionic, or complex. Those of real or quaternionic type are self-conjugate, while those of complex type are not. There is a set $B(G)$ of irreducible representations called basic such that $R(G)$ is a polynomial algebra on $B(G)$. If $\rho$ is any representation, let

$$
\widetilde{\rho}=\rho-\operatorname{dim}(\rho) \in I(G) .
$$

Let $Q, Q_{\mathbf{R}}$, and $Q_{\mathbf{H}}$ be as in 2.2 and its preamble. Then $Q$ is a free abelian group with basis $\{\widetilde{\rho}: \rho \in B(G)\}$. The set $B(G)$ can be partitioned into subsets $B_{\mathbf{R}}(G), B_{\mathbf{H}}(G)$, and $B_{\mathbf{C}}(G)$ of representations of real, quaternionic, and complex type, respectively. The set $B_{\mathbf{C}}(G)$ is composed of pairs of conjugate representations. Let $B_{\mathbf{C}}^{\prime}(G)$ contain one element from each pair of conjugate elements of $B_{\mathbf{C}}(G)$. Then $Q_{\mathbf{R}}$ is a free abelian group with basis

$$
\left\{\widetilde{\rho}: \rho \in B_{\mathbf{R}}(G)\right\} \cup\left\{2 \widetilde{\rho}: \rho \in B_{\mathbf{H}}(G)\right\} \cup\left\{\widetilde{\rho}+t(\widetilde{\rho}): \rho \in B_{\mathbf{C}}^{\prime}(G)\right\},
$$

and similarly for $Q_{\mathbf{H}}$ with $\mathbf{R}$ and $\mathbf{H}$ interchanged.

Now we state Bousfield's Technical Condition.

Definition 2.4. Let $G$ be a simply-connected compact Lie group, and let

$$
H(G)=\operatorname{ker}(1-t) / \operatorname{im}(1+t) .
$$

Then $H(G)$ is an augmented $\mathbf{Z} / 2$-graded polynomial algebra over $\mathbf{Z} / 2$ on

$$
B_{\mathbf{R}}(G) \cup B_{\mathbf{H}}(G) \cup\left\{\rho t(\rho): \rho \in B_{\mathbf{C}}^{\prime}(G)\right\},
$$


where $\operatorname{gr}\left(B_{\mathbf{R}}(G)\right)=0, \operatorname{gr}\left(B_{\mathbf{H}}(G)\right)=1$, and $\operatorname{gr}(\rho t(\rho))=0$, while the augmentation $\epsilon$ satisfies $\epsilon(\rho)=\operatorname{dim}(\rho) \bmod 2$. Let $H_{\mathbf{R}}(G)$ denote the subgroup of $H(G)$ of grading 0 . There is an augmentation-preserving algebra homomorphism $\phi: R(G) \rightarrow H(G)$ defined by $\phi(\rho)=\rho t(\rho)$. The image of $\phi$ is contained in $H_{\mathbf{R}}(G)$. This $\phi$ induces a morphism of indecomposables

$$
\bar{\phi}: I(R(G)) / I^{2}(R(G)) \rightarrow I(H(G)) / I^{2}(H(G)),
$$

whose image lies in the summand

$$
I\left(H_{\mathbf{R}}(G)\right) / I^{2}(H(G)):=\operatorname{Ind}_{\mathbf{R}}(H(G))
$$

of grading 0. The morphism $\bar{\phi}$ preserves Adams operations, and its image is automatically a $\psi^{3}$-submodule of $\operatorname{Ind}_{\mathbf{R}}(H(G))$. We say that $G$ satisfies the Technical Condition if $\operatorname{im}(\bar{\phi})$ is a direct summand of $\operatorname{Ind}_{\mathbf{R}}(H(G))$ as a $\psi^{3}$-module; i.e., if it has a complementary $\psi^{3}$-submodule.

In e-mails dated January 30, 2003, and February, 8, 2003, Bousfield wrote that he has a proof of the following result, which he is in the process of writing. In fact, he will prove more; the author has just extracted from various letters from Bousfield the portion of these consequences necessary for the specific applications to $v_{1}$-periodic homotopy groups.

Theorem 2.5. (Bousfield) If $G$ satisfies the Technical Condition, then Conjecture 2.2 is true for $G$.

In those same e-mails, Bousfield wrote that he has an idea of how he might be able to prove Conjecture 2.2 without assuming the Technical Condition, but that this is more speculative.

\section{Compact simple Lie groups and the Technical Condition}

In this section, we prove Theorem 1.3, which states exactly which of the simplyconnected compact simple Lie groups satisfy the Technical Condition 2.4.

We begin by tabulating for the compact simple Lie groups a set of basic representations and their division into types. For the classical groups, this information is proved in [11, VI], while for the exceptional groups it is extracted from [17]. 
Table 3.1. Types of basic representations

\begin{tabular}{l|l|l|l|l|}
\multicolumn{1}{c|}{$G$} & \multicolumn{1}{|c|}{$B_{\mathbf{R}}(G)$} & $B_{\mathbf{H}}(G)$ & $B_{\mathbf{C}}^{\prime}(G)$ & $t\left(B_{\mathbf{C}}^{\prime}(G)\right)$ \\
\hline$S U(2 n+1)$ & & & $\lambda_{1}, \ldots, \lambda_{n}$ & $t\left(\lambda_{i}\right)=\lambda_{2 n+1-i}$ \\
\hline$S U(2 n), n$ even & $\lambda_{n}$ & & $\lambda_{1}, \ldots, \lambda_{n-1}$ & $t\left(\lambda_{i}\right)=\lambda_{2 n-i}$ \\
\hline$S U(2 n), n$ odd & & $\lambda_{n}$ & $\lambda_{1}, \ldots, \lambda_{n-1}$ & $t\left(\lambda_{i}\right)=\lambda_{2 n-i}$ \\
\hline$S p(n)$ & $\begin{array}{l}\lambda_{i}, i \text { even, } \\
2 \leqslant i \leqslant n\end{array}$ & $\begin{array}{l}\lambda_{i}, i \text { odd, } \\
1 \leqslant i \leqslant n\end{array}$ & & \\
\hline $\begin{array}{l}\text { Spin }(2 n+1), \\
n \equiv 0,3 \bmod 4\end{array}$ & $\lambda_{1}, \ldots, \lambda_{n-1}$, & & & \\
\hline $\begin{array}{l}\text { Spin }(2 n+1), \\
n \equiv 1,2 \bmod 4\end{array}$ & $\lambda_{1}, \ldots, \lambda_{n-1}$ & $\Delta$ & & \\
\hline $\operatorname{Spin}(2 n), n$ odd & $\lambda_{1}, \ldots, \lambda_{n-2}$ & & $\Delta_{+}$ & $t\left(\Delta_{+}\right)=\Delta \Delta_{-}$ \\
\hline $\begin{array}{l}\operatorname{Spin}(2 n), \\
n \equiv 0 \bmod 4\end{array}$ & $\lambda_{1}, \ldots, \lambda_{n-2}$, & & & \\
\hline $\begin{array}{l}\text { Spin }(2 n), \\
n \equiv 2 \bmod 4\end{array}$ & $\Delta_{1}, \ldots, \lambda_{n-2}$ & $\Delta_{+}, \Delta_{-}$ & & \\
\hline$G_{2}, F_{4}, E_{8}$ & $\rho_{1}, \ldots, \rho_{t}$ & & & $t\left(\rho_{1}\right)=\rho_{6}$, \\
\hline$E_{6}$ & $\rho_{2}, \rho_{4}$ & & $\rho_{1}, \rho_{3}$ & \\
\hline$E_{7}$ & $\rho_{1}, \rho_{3}, \rho_{4}, \rho_{6}$ & $\rho_{2}, \rho_{5}, \rho_{7}$ & & \\
\hline
\end{tabular}

We will say more about the specifics of the basic representations $\rho_{i}$ of $E_{6}$ and $E_{7}$ when we make specific applications later.

We begin with an elementary proposition and two corollaries.

Proposition 3.2. In the notation of Definition 2.4, $\operatorname{Ind}_{\mathbf{R}}(H(G))$ is a vector space over $\mathbf{Z} / 2$ with basis $\widetilde{B}_{\mathbf{R}}(G) \cup B(\bar{\phi})$, where $\widetilde{B}_{\mathbf{R}}(G)=\left\{\widetilde{\rho}: \rho \in B_{\mathbf{R}}(G)\right\}$, and $B(\bar{\phi})=$ $\left\{\widetilde{\rho} \cdot t(\widetilde{\rho}): \rho \in B_{\mathbf{C}}^{\prime}(G)\right\}$ is a basis for $\operatorname{im}(\bar{\phi})$.

Proof. This follows immediately from the definitions.

Corollary 3.3. If either $B_{\mathbf{R}}(G)$ or $B_{\mathbf{C}}^{\prime}(G)$ is empty, then $G$ satisfies the Technical Condition.

Proof. If $B_{\mathbf{R}}(G)$ is empty, then $\operatorname{im}(\bar{\phi})=\operatorname{Ind}_{\mathbf{R}}(H(G))$, while if $B_{\mathbf{C}}^{\prime}(G)$ is empty, then $\operatorname{im}(\bar{\phi})=0$, both of which are clearly $\psi^{3}$-direct summands of $\operatorname{Ind}_{\mathbf{R}}(H(G))$.

Corollary 3.4. A simply-connected compact simple Lie group which does not equal $S U(4 m)$, Spin $(4 k+2)$, or $E_{6}$ satisfies the Technical Condition.

Proof. By Table 3.1, all other simply-connected compact simple Lie groups satisfy the hypothesis of Corollary 3.3.

We handle the three (families of) Lie groups not covered by Corollary 3.4 in separate theorems, 3.5, 3.7, and 3.32. 
Theorem 3.5. For each $m \geqslant 1, S U(4 m)$ satisfies the Technical Condition.

Proof. Referring to Table 3.1, we see that

$$
\operatorname{im}(\bar{\phi})=\left\langle\widetilde{\lambda}_{i} \widetilde{\lambda}_{4 m-i}: 1 \leqslant i<2 m\right\rangle .
$$

We will prove

$$
\text { the coefficient of } \widetilde{\lambda}_{i} \widetilde{\lambda}_{4 m-i} \text { in } \psi^{3}\left(\widetilde{\lambda}_{2 m}\right) \text { is even, }
$$

which, with 3.2 , will imply the theorem, since $\left\langle\widetilde{\lambda}_{2 m}\right\rangle$ is then a $\psi^{3}$-submodule of $\operatorname{Ind}_{\mathbf{R}}(H(S U(4 m)))$ complementary to $\operatorname{im}(\bar{\phi})$.

Let $T$ denote the maximal torus of $S U(4 \mathrm{~m})$. Then

$$
R(T)=\mathbf{Z}\left[x_{1}, \ldots, x_{4 m}\right] /\left(x_{1} \cdots x_{4 m}-1\right),
$$

and $R(S U(4 m)) \rightarrow R(T)$ is a ring homomorphism sending $\lambda_{k}$ to $\sigma_{k}$, and sending $\psi^{3}\left(\lambda_{2 m}\right)$ to $\sum x_{i_{1}}^{3} \cdots x_{i_{2 m}}^{3}$, which we call $p$. Here the sum is over all $i_{1}<\cdots<i_{2 m}$, and $\sigma_{k}$ is the $k$ th elementary symmetric polynomial in $x_{1}, \cdots, x_{4 m}$.

This $p$ can be written as an integer polynomial $f\left(\sigma_{1}, \ldots, \sigma_{4 m}\right)$ in which each term has grading $6 m$, where $\operatorname{gr}\left(\sigma_{i}\right)=i$. Then $\psi^{3}\left(\lambda_{2 m}\right)$ is the same polynomial $f\left(\lambda_{1}, \cdots, \lambda_{4 m}\right)$. To write $\psi^{3}\left(\widetilde{\lambda}_{2 m}\right)$ in terms of $\widetilde{\lambda}_{1}, \ldots, \widetilde{\lambda}_{4 m-1}$, we replace each $\lambda_{i}$ by $\widetilde{\lambda}_{i}+\left(\begin{array}{c}4 m \\ i\end{array}\right)$. Note $\widetilde{\lambda}_{4 m}=0$.

Let $i<2 m$, and let $\tau:=\lambda_{1}^{j_{1}} \cdots \lambda_{4 m}^{j_{4 m}}$ have odd coefficient in $f\left(\lambda_{1}, \ldots, \lambda_{4 m}\right)$. Then

$$
\tau=\left(\widetilde{\lambda}_{1}+\left(\begin{array}{c}
4 m \\
1
\end{array}\right)\right)^{j_{1}} \cdots\left(\widetilde{\lambda}_{4 m}+\left(\begin{array}{c}
4 m \\
4 m
\end{array}\right)\right)^{j_{4 m}}
$$

contains a term $\beta \widetilde{\lambda}_{i} \widetilde{\lambda}_{4 m-i}$ with $\beta \in \mathbf{Z}$ if and only if $j_{i}>0$ and $j_{4 m-i}>0$.

If we write $\tau=\lambda_{i} \lambda_{4 m-i} \prod \lambda_{k_{\ell}}$, then the coefficient $\beta$ of $\widetilde{\lambda}_{i} \widetilde{\lambda}_{4 m-i}$ in $\tau$ is equal to $j_{i} j_{4 m-i} \prod\left(\begin{array}{c}4 m \\ k_{\ell}\end{array}\right)$. Note that $k_{\ell}$ may be repeated, and may equal $i$ or $4 m-i$, but $\sum k_{\ell}=6 m-4 m=2 m$. Now we note that if $\prod\left(\begin{array}{c}4 m \\ k_{\ell}\end{array}\right)$ is odd, then each $\left(\begin{array}{c}4 m \\ k_{\ell}\end{array}\right)$ is odd, hence each $k_{\ell}$ is at least as 2-divisible as $4 m$, and so $2 m=\sum \sum_{\mathcal{C}_{\ell}}$ is at least as 2-divisible as $4 m$, which is impossible. Thus the coefficient of $\widetilde{\lambda}_{i} \widetilde{\lambda}_{4 m-i}$ is even, proving (3.6).

The proof of the next result involves more combinatorics.

Theorem 3.7. $\operatorname{Spin}(4 k+2)$ satisfies the Technical Condition if and only if $k$ is a 2-power.

Proof. It is well-known (see, e.g., [18, p.151]) that $j^{*}: R(S U(2 n)) \rightarrow R(\operatorname{Spin}(2 n))$ satisfies

$$
j^{*}\left(\lambda_{i}\right)=j^{*}\left(\lambda_{2 n-i}\right) .
$$

Let $\mu_{i}=j^{*}\left(\lambda_{i}\right)$. Then (see, e.g., [11, VI.6.2]) $R(\operatorname{Spin}(2 n))$ has basic representations $\mu_{1}, \ldots, \mu_{n-2}, \Delta_{+}, \Delta_{-}$with

$$
\begin{gathered}
\Delta_{+} \Delta_{-}=\mu_{n-1}+\mu_{n-3}+\mu_{n-5}+\cdots \\
\Delta_{+}^{2}+\Delta_{-}^{2}=\mu_{n}+2\left(\mu_{n-2}+\mu_{n-4}+\cdots\right) .
\end{gathered}
$$

By 3.1, $\widetilde{B}_{\mathbf{R}}(\operatorname{Spin}(2 n))=\left\{\widetilde{\mu}_{1}, \ldots, \widetilde{\mu}_{n-2}\right\}$, and $B(\bar{\phi})=\{P\}$, where $P:=\widetilde{\Delta}_{+} \widetilde{\Delta}_{-}$. The theorem follows from the following result, in which $n=2 k+1$. 
Lemma 3.11. Let $\bar{\epsilon}=\left(\epsilon_{1}, \ldots, \epsilon_{2 k-1}\right)$ with $\epsilon_{i} \in \mathbf{Z} / 2$. Let $W(\bar{\epsilon})$ denote the subspace of the $\mathbf{Z} / 2$-vector space $\operatorname{Ind}_{\mathbf{R}}(H(\operatorname{Spin}(4 k+2)))$ spanned by $\left\{\widetilde{\mu}_{1}+\epsilon_{1} P, \ldots, \widetilde{\mu}_{2 k-1}+\right.$ $\left.\epsilon_{2 k-1} P\right\}$. Every $\mathbf{Z} / 2$-subspace complementary to $\operatorname{im}(\bar{\phi})=\langle P\rangle$ is of this form.

1. If $k$ is a 2-power, then $W(\overline{0})$ is a $\psi^{3}$-submodule of $\operatorname{Ind}_{\mathbf{R}}(H(\operatorname{Spin}(4 k+2)))$, while

2. if $k$ is not a 2-power, $W(\bar{\epsilon})$ can never be a $\psi^{3}$-submodule of $\operatorname{Ind}_{\mathbf{R}}(H(\operatorname{Spin}(4 k+$ $2))$ ).

In order to prove Lemma 3.11, we need an explicit formula for $\psi^{3}$ on the basis $\left\{\widetilde{\lambda}_{1}, \ldots, \widetilde{\lambda}_{2 n-1}\right\}$ of the free abelian group

$$
Q(S U(4 k+2)):=I(R(S U(4 k+2))) / I^{2}(R(S U(4 k+2))) .
$$

This will then be transported to $\operatorname{Ind}_{\mathbf{R}}(H(\operatorname{Spin}(4 k+2)))$ using $j^{*},(3.8)$, and (3.9).

Theorem 3.12. Define integers $c_{n, \ell}^{k}$ by

$$
\left(1+x+\cdots+x^{k-1}\right)^{n}=\sum c_{n, \ell}^{k} x^{\ell} .
$$

In $Q(S U(n))$,

$$
\psi^{k}\left(\widetilde{\lambda}_{i}\right)=k \sum_{\ell \geqslant 0}(-1)^{k i+i+\ell} c_{n, \ell}^{k} \widetilde{\lambda}_{k i-\ell}
$$

Proof. Let $\beta: I(R(G)) / I^{2}(R(G)) \rightarrow P K^{-1}(G)$ be Hodgkin's isomorphism ([16]), where $P$ denotes the primitives. As in [2, pp. 42-43], let $B_{i} \in P K^{1}(S U(n))$ correspond to $\beta\left(\widetilde{\lambda}_{i}\right)$ under Bott periodicity. We will prove that

$$
\psi^{k}\left(B_{i}\right)=\sum_{\ell \geqslant 0}(-1)^{k i+i+\ell} c_{n, \ell}^{k} B_{k i-\ell} .
$$

Then (3.14) follows from the fact that $\psi^{k}$ in $K^{1}(G)$ corresponds to $\psi^{k} / k$ in $K^{-1}(G)$.

In $[2,3.2]$, it is shown that $B_{j}=\sum(-1)^{\ell+1}\left(\begin{array}{c}n \\ j-\ell\end{array}\right) \xi_{\ell}$, where $\xi_{\ell}=\xi^{\ell}-1$ satisfies $\psi^{k}\left(\xi_{\ell}\right)=\xi_{k \ell}$. Thus it suffices to prove

$$
\sum_{\ell}(-1)^{\ell+1}\left(\begin{array}{c}
n \\
i-\ell
\end{array}\right) \xi_{k \ell}=\sum_{\ell}(-1)^{k i+i+\ell} c_{n, \ell}^{k} \sum_{t}(-1)^{t+1}\left(\begin{array}{c}
n \\
k i-\ell-t
\end{array}\right) \xi_{t},
$$

where the sums are taken over all values which give meaningful terms. Note that there are relations among the $\xi_{i}$ 's when $i \geqslant n$, but they are the same on both sides of the equation, and hence need not be considered. The $\xi_{i}$ 's are just formal variables, and so can be replaced by $x^{-i}$. Thus we wish to prove

$$
\left.\sum_{\ell}(-1)^{\ell}{ }_{i-\ell}^{n}\right) x^{-k \ell}=\sum_{\ell}(-1)^{k i+i+\ell} c_{n, \ell}^{k} x^{\ell} \sum_{t}(-1)^{t}\left(\begin{array}{c}
n \\
k i-\ell-t
\end{array}\right) x^{-\ell-t} .
$$

Multiplying both sides by $(-1)^{i} x^{k i}$, it is equivalent to show

$$
\sum_{\ell}(-1)^{i-\ell}\left(\begin{array}{c}
n \\
i-\ell
\end{array}\right)\left(x^{k}\right)^{i-\ell}=\sum_{\ell} c_{n, \ell}^{k} x^{\ell} \sum_{t}(-1)^{k i-\ell-t}\left(\begin{array}{c}
n \\
k i-\ell-t
\end{array}\right) x^{k i-\ell-t} .
$$

The left hand side equals $\left(1-x^{k}\right)^{n}$, while the right hand side equals $(1+x+\cdots+$ $\left.x^{k-1}\right)^{n}(1-x)^{n}$, and these are equal, establishing (3.15). 
Remark 3.16. Another approach to Theorem 3.12 is illustrated by

$$
\psi^{2}\left(\widetilde{\lambda}_{1}\right)=-2 \widetilde{\lambda}_{2}+2 n \widetilde{\lambda}_{1}+\widetilde{\lambda}_{1}^{2}
$$

in $R(S U(n))$, which is readily verified to be consistent with one case of 3.12.

Proof. Using $R(S U(n)) \rightarrow R(T)$ as in the proof of $3.5, \widetilde{\lambda}_{1}$ corresponds to $x_{1}+$ $\cdots+x_{n}-n$, while $\widetilde{\lambda}_{2}$ corresponds to $\sum_{i<j} x_{i} x_{j}-\left(\begin{array}{c}n \\ 2\end{array}\right)$. Then $\psi^{2}\left(\widetilde{\lambda}_{1}\right)$ corresponds to

$$
x_{1}^{2}+\cdots+x_{n}^{2}-n=\left(x_{1}+\cdots+x_{n}\right)^{2}-n-2 \sum_{i<j} x_{i} x_{j},
$$

which corresponds to $\left(\widetilde{\lambda}_{1}+n\right)^{2}-n-2\left(\widetilde{\lambda}_{2}+\left(\begin{array}{c}n \\ 2\end{array}\right)\right)=\widetilde{\lambda}_{1}^{2}+2 n \widetilde{\lambda}_{1}-2 \widetilde{\lambda}_{2}$.

Proof of Lemma 3.11. We work in the $\mathbf{Z} / 2$-vector space $V:=\operatorname{Ind}_{\mathbf{R}}(H(\operatorname{Spin}(4 k+$ $2))$ ). Since $\operatorname{dim}\left(\Delta_{ \pm}\right)$is even, (3.9) becomes

$$
P=\widetilde{\mu}_{2 k}+\widetilde{\mu}_{2 k-2}+\cdots .
$$

Since $\Delta_{+}^{2}+\Delta_{-}^{2}=(1+t)\left(\Delta_{+}^{2}\right),(3.10)$ implies $\widetilde{\mu}_{2 k+1}=0$ in $V$. ¿From (3.14) and (3.8), we obtain

$$
\psi^{3}\left(\widetilde{\mu}_{j}\right)=\sum c_{4 k+2,3 j-\ell}^{3} \widetilde{\mu}_{\ell}=\sum_{\ell=1}^{2 k}\left(c_{4 k+2,3 j-\ell}^{3}+c_{4 k+2,3 j-(4 k+2-\ell)}^{3}\right) \widetilde{\mu}_{\ell} .
$$

We will use $(3.17)$ to express $\widetilde{\mu}_{2 k}$ as $P-\widetilde{\mu}_{2 k-2}-\widetilde{\mu}_{2 k-4}-\cdots$.

By (3.13), since we work $\bmod 2$,

$$
c_{4 k+2, i}^{3}= \begin{cases}c_{2 k+1, i^{\prime}}^{3} & \text { if } i=2 i^{\prime} \\ 0 & \text { if } i \text { odd }\end{cases}
$$

Thus (3.18) implies that there is a splitting as $\psi^{3}$-modules,

$$
V=V_{\mathrm{od}} \oplus V_{\mathrm{ev}}
$$

where $V_{\text {od }}=\left\langle\widetilde{\mu}_{2 i-1}: 1 \leqslant i \leqslant k\right\rangle$ and $V_{\text {ev }}=\left\langle P, \widetilde{\mu}_{2 i}: 1 \leqslant i \leqslant k-1\right\rangle$. Thus $\operatorname{im}(\bar{\phi})=$ $\langle P\rangle$ has a complementary $\psi^{3}$-submodule in $V$ if and only if it has a complementary $\psi^{3}$-submodule in $V_{\text {ev }}$, and so we focus our attention on the latter.

Let $v_{i}:=\widetilde{\mu}_{2 i} \in V_{\mathrm{ev}}$ and $c_{i}:=c_{2 k+1, i}^{3}$. Then (3.18) becomes

$$
\psi^{3}\left(v_{j}\right)=\sum_{t=1}^{k-1}\left(c_{3 j-t}+c_{3 j-2 k-1+t}\right) v_{t}+\left(c_{3 j-k}+c_{3 j-k-1}\right)\left(P+v_{k-1}+v_{k-2}+\cdots\right) .
$$

One can use these sorts of formulas to prove $\psi^{3}(P)=P$, but this also follows from the fact that $\operatorname{im}(\bar{\phi})$ is a $\psi^{3}$-submodule by naturality.

Now we can easily prove $3.11(1)$. If $k=2^{e}$, then $c_{i}=1$ implies $i=0,1$, or 2 , or $i \geqslant 2^{e+1}$. By (3.19), we must prove that if $1 \leqslant j \leqslant k-1$, then $c_{3 j-k}+c_{3 j-k-1}=0$. Note that for such $j$, we have $3 j-k \leqslant 2 k-3<2^{e+1}$. Thus the only way to have $c_{3 j-k}+c_{3 j-k-1}=1$ is if $3 j-k=0$ or 3 . But this is impossible, since $k \neq \equiv \bmod 3$.

To prove part $(2)$, let

$$
B=\left(\begin{array}{ll}
A & \overline{0} \\
\bar{r} & 1
\end{array}\right)
$$


denote the matrix of $\psi^{3}$ with respect to the ordered basis $\left\{v_{1}, \ldots, v_{k-1}, P\right\}$. Thus $A=\left(a_{i, j}\right)$ is a $(k-1)$-by- $(k-1)$ matrix. The last column of $B$ is due to $\psi^{3}(P)=P$, observed earlier. We will prove

a. If $k$ is not a 2-power, then $\bar{r} \neq 0$.

b. $A+I$ is singular.

Lemma 3.11(2) then follows. Indeed, suppose $k$ is not a 2-power and $W(\bar{\epsilon})$ is a $\psi^{3}$-submodule. Then, for $1 \leqslant j \leqslant k-1$,

$$
\begin{aligned}
\psi^{3}\left(v_{j}+\epsilon_{j} P\right) & =\sum_{i=1}^{k-1} a_{i, j} v_{i}+r_{j} P+\epsilon_{j} P \\
& =\sum_{i=1}^{k-1} a_{i, j}\left(v_{i}+\epsilon_{i} P\right)+\left(\sum_{i=1}^{k-1} a_{i, j} \epsilon_{i}+r_{j}+\epsilon_{j}\right) P .
\end{aligned}
$$

The coefficient of $P$ must be 0 for each $j$. Thus, with $(-)^{T}$ denoting the transpose,

$$
(A+I) \bar{\epsilon}^{T}=\bar{r}^{T},
$$

and since $\bar{r} \neq 0$ by (a), $A+I$ must be nonsingular, contradicting (b).

It remains to prove (a) and (b). Part (a) follows from (3.19) and Lemma 3.20, while (b) follows from Lemma 3.24.

Lemma 3.20. Let $\left(1+x+x^{2}\right)^{2 k+1}=\sum c_{i} x^{i}$. If $k$ is not a 2-power, there exists $j$ satisfying $1 \leqslant j \leqslant k-1$ and $c_{3 j-k}+c_{3 j-k-1} \equiv 1 \bmod 2$.

Proof. We write $k=2^{e} u$ with $u>1$ odd, and divide into cases depending on the $\bmod 6$ value of $u$.

Case 1. If $u=3 a$, then $j=2^{e} a$ works, since $c_{0}+c_{-1}=1$.

Case 2. If $u=6 a+1$, then $j=2^{e}(2 a+1)$ works. (Note that if $a=0$, then $j=k$, explaining the failure of the lemma when $k$ is a 2-power.) To see this, note that

$$
\left(1+x+x^{2}\right)^{2 k+1} \equiv 1+x+x^{2}+x^{2^{e+1}} \bmod \left(x^{2^{e+1}+1}\right),
$$

and hence $c_{2^{e+1}}+c_{2^{e+1}-1}=1$.

Case 3. If $u=3 \cdot 2^{f} \alpha-1$ with $f \geqslant 1$ and $\alpha$ odd, then $j=2^{e}\left(2^{f} \alpha+2^{f}-1\right)$ works. To see this, let $c(p, i)$ denote the coefficient of $x^{i}$ in the polynomial or power series $p=p(x)$. Then $c_{3 j-k}+c_{3 j-k-1}$ equals

$$
c\left(\left(1+x+x^{2}\right)^{2 k+1}, 3 \cdot 2^{e+f}-2^{e+1}\right)+c\left(\left(1+x+x^{2}\right)^{2 k+1}, 3 \cdot 2^{e+f}-2^{e+1}-1\right) .
$$

$\operatorname{Mod} x^{2^{e+f+2}}$, we have

$$
\left(1+x+x^{2}\right)^{2 k+1} \equiv\left(1+x+x^{2}\right)\left(1+x^{2^{e+f+1}}\right)\left(1+x+x^{2}\right)^{-2^{e+1}} .
$$

Thus (3.21) equals

$$
\begin{aligned}
& c\left(g, 3 \cdot 2^{e+f}-2^{e+1}\right)+c\left(g, 3 \cdot 2^{e+f}-2^{e+1}-1\right) \\
+ & c\left(g, 2^{e+f}-2^{e+1}\right)+c\left(g, 2^{e+f}-2^{e+1}-1\right),
\end{aligned}
$$

where

$$
g=\left(1+x+x^{2}\right)\left(1+x^{2^{e+1}}+x^{2^{e+2}}\right)^{-1}=\left(1+x+x^{2}\right) \sum_{i \geqslant 0}\left(x^{3 i 2^{e+1}}+x^{2^{e+1}(3 i+1)}\right) .
$$


Here we have used ( $\bmod 2$, as always)

$$
\left(1+z+z^{2}\right)^{-1}=\frac{1+z}{1+z^{3}}=\sum_{i \geqslant 0}\left(z^{3 i}+z^{3 i+1}\right) .
$$

If $e=0$, then $g=\sum_{j \geqslant 0}\left(x^{3 j}+x^{3 j+1}\right)$, and so $c\left(g, 3 \cdot 2^{f}-2\right)=c\left(g, 3 \cdot 2^{f}-3\right)=1$, while $c\left(g, 2^{f}-2\right)+c\left(g, 2^{f}-3\right)=1$ since $2^{f}-2 \not \equiv 1 \bmod 3$. Thus (3.22) equals 1 in this case. If $e>0, c(g, j)=1$ if and only if $j \equiv 0,1,2,2^{e+1}, 2^{e+1}+1$, or $2^{e+1}+2$ $\bmod 3 \cdot 2^{e+1}$. The $\bmod 3 \cdot 2^{e+1}$ values of the four exponents of $x$ in $(3.22)$ are $-2^{e+1}$, $-2^{e+1}-1,0$ or $2^{e+1}$, and -1 or $2^{e+1}-1$. Hence the third coefficient is 1 while the others are 0 .

Like the above lemma, Lemmas 3.24, 3.30, and 3.31 below all deal with mod 2 polynomials.

Lemma 3.24. Let $\left(1+x+x^{2}\right)^{2 k+1}=\sum c_{i} x^{i}$. Let $A=\left(a_{i, j}\right)$ be the $(k-1)$-by- $(k-1)$ matrix with

$$
a_{i, j}=c_{3 j-i}+c_{3 j-2 k-1+i}+c_{3 j-k}+c_{3 j-k-1} .
$$

Then $A+I$ is singular.

Proof. Let $\gamma_{j}=1$ if, for $e \geqslant 0$,

$$
\begin{cases}k=2^{e}(4 m-1) & \text { and } 1 \leqslant\left(j \bmod 2^{e+2}\right) \leqslant 2^{e+1} \\ k=2^{e}(4 m+1), m>0, & \text { and } 3 \cdot 2^{e} \leqslant\left(k-j \bmod 2^{e+3}\right) \leqslant 5 \cdot 2^{e}-1, \\ k=2^{e+1} & \text { and } 1 \leqslant j \leqslant 2^{e}\end{cases}
$$

and $\gamma_{j}=0$ otherwise. We will show that, for $1 \leqslant i \leqslant k-1$,

$$
\left(\sum_{j=1}^{k-1} \gamma_{j} a_{i, j}\right)+\gamma_{i}=0
$$

which establishes the linear dependence of some of the columns of $A+I$. Observing that $\gamma_{k}=0$, it is equivalent to show that, for $1 \leqslant i \leqslant k$,

$$
\sum_{j=1}^{k-1} \gamma_{j}\left(c_{3 j-i}+c_{3 j-2 k-1+i}\right)=\gamma_{i}
$$

We will, in fact, prove that (3.25) is true for all $i \geqslant 1$.

We begin with the case $k=2^{e}(4 m-1)$. Since $k \equiv-2^{e} \bmod 2^{e+2}$, we have

$$
\begin{aligned}
\sum_{j=1}^{k-1} \gamma_{j} c_{3 j-i} & =\sum_{\ell \geqslant 0} \sum_{d=0}^{2^{e+1}-1} c_{3\left(k-2^{e}-d-\ell 2^{e+2}\right)-i} \\
& =c\left(g_{k}(x), 3\left(k-2^{e}\right)-i\right),
\end{aligned}
$$

where

$$
g_{k}(x)=\left(1+x+x^{2}\right)^{2 k+1} \frac{1+x^{3}+\cdots+x^{3\left(2^{e+1}-1\right)}}{1+x^{3 \cdot 2^{e+2}}}
$$


and $c(-,-)$ is as in the previous proof. Similarly,

$$
\sum_{j=1}^{k-1} \gamma_{j} c_{3 j-2 k-1+i}=c\left(g_{k}(x), k-3 \cdot 2^{e}-1+i\right) .
$$

Thus we must show

$$
\begin{aligned}
& c\left(g_{k}(x), 3\left(2^{e+2} m-2^{e+1}\right)-i\right)+c\left(g_{k}(x), 2^{e+2} m-2^{e+2}+i-1\right) \\
= & \begin{cases}1 & \text { if } 1 \leqslant\left(i \quad \bmod 2^{e+2}\right) \leqslant 2^{e+1} \\
0 & \text { otherwise. }\end{cases}
\end{aligned}
$$

We have

$$
\begin{aligned}
g_{k}(x) & =\left(1+x+x^{2}\right)^{2 k+1} \frac{1+x^{3 \cdot 2^{e+1}}}{\left(1+x^{3}\right)\left(1+x^{3 \cdot 2^{e+2}}\right)} \\
& =\left(1+x+x^{2}\right)^{2 k+2^{e+1}} \frac{(1+x)^{2^{e+1}}-1}{1+x^{3 \cdot 2^{e+2}}} \\
& =\left(1+x^{2^{e+3}}+x^{2^{e+4}}\right)^{m} \frac{(1+x)^{2^{e+1}}-1}{1+x^{3 \cdot 2^{e+2}}} .
\end{aligned}
$$

If $2^{e+1}+1 \leqslant\left(i \bmod 2^{e+2}\right) \leqslant 2^{e+2}$, then both exponents of $x$ in $(3.26)$ are in the mod $2^{e+2}$ range from $2^{e+1}$ up to $2^{e+2}-1$. Such terms have coefficient 0 in $g_{k}(x)$, since it is of the form $f\left(x^{2^{e+2}}\right) \sum_{\ell=0}^{2^{e+1}-1} x^{\ell}$. Thus the "otherwise" part of (3.26) is verified.

Now let $i=2^{e+2} t+\epsilon$ with $1 \leqslant \epsilon \leqslant 2^{e+1}$. The left hand side of (3.26) equals

$$
c\left(p_{1}(x), 3 \cdot 2^{e+2} m-2 \cdot 2^{e+2}-2^{e+2} t\right)+c\left(p_{1}(x), 2^{e+2} m-2^{e+2}+2^{e+2} t\right),
$$

where

$$
p_{1}(x)=\frac{\left(1+x^{2^{e+3}}+x^{2^{e+4}}\right)^{m}}{1+x^{3 \cdot 2^{e+2}}}
$$

because the $(1+x)^{2^{e+1}-1}$ in $g_{k}(x)$ corresponds to the range of values of $i$. Replacing $x^{2^{e+2}}$ by $x$, that (3.27) has the desired value of 1 follows from Lemma 3.30. (Note $i$ in 3.30 corresponds to $3 m-2-t$ above.)

The proof when $k=2^{e}(4 m+1)$ and $m>0$ is similar. We now have

$$
\begin{aligned}
\sum_{j=1}^{k-1} \gamma_{j} c_{3 j-i} & =\sum_{\ell \geqslant 0} \sum_{d=0}^{2^{e+1}-1} c_{3\left(k-3 \cdot 2^{e}-d-\ell 2^{e+3}\right)-i} \\
& =c\left(h_{k}(x), 3\left(k-3 \cdot 2^{e}\right)-i\right),
\end{aligned}
$$

where

$$
\begin{aligned}
h_{k}(x) & =\left(1+x+x^{2}\right)^{2 k+1} \frac{1+x^{3}+\cdots+x^{3\left(2^{e+1}-1\right)}}{1+x^{3 \cdot 2^{e+3}}} \\
& =\left(1+x^{2^{e+3}}+x^{2^{e+4}}\right)^{m}\left(1+x^{2^{e+2}}+x^{2^{e+3}}\right) \frac{(1+x)^{2^{e+1}-1}}{1+x^{3 \cdot 2^{e+3}}},
\end{aligned}
$$


and similarly

$$
\sum_{j=1}^{k-1} \gamma_{j} c_{3 j-2 k-1+i}=c\left(h_{k}(x), k-9 \cdot 2^{e}-1+i\right) .
$$

Thus we must show

$$
\begin{aligned}
& c\left(h_{k}(x), 3\left(2^{e+2} m-2^{e+1}\right)-i\right)+c\left(h_{k}(x), 2^{e+2} m-2^{e+3}-1+i\right) \\
= & \begin{cases}1 & \text { if } i=2^{e+2} t+\epsilon, 1 \leqslant \epsilon \leqslant 2^{e+1}, m+t \text { odd } \\
0 & \text { otherwise. }\end{cases}
\end{aligned}
$$

If $2^{e+1}+1 \leqslant\left(i \bmod 2^{e+2}\right) \leqslant 2^{e+2}$, then both exponents in (3.28) are in a range $\left(2^{e+1}\right.$ to $\left.2^{e+2}-1 \bmod 2^{e+2}\right)$ where all coefficients of $h_{k}(x)$ are 0 . If, on the other hand, $i=2^{e+2} t+\epsilon$ with $1 \leqslant \epsilon \leqslant 2^{e+1}$, then the left hand side of (3.28) equals

$$
c\left(p_{2}(x), 3 \cdot 2^{e+2} m-2^{e+2} t-2^{e+3}\right)+c\left(p_{2}(x), 2^{e+2} m-2^{e+3}+2^{e+2} t\right)
$$

with

$$
p_{2}(x)=\frac{\left(1+x^{2^{e+3}}+x^{2^{e+4}}\right)^{m}\left(1+x^{2^{e+2}}+x^{2^{e+3}}\right)}{1+x^{3 \cdot 2^{e+3}}} .
$$

Replacing $x^{2^{e+2}}$ by $x$, and letting $\ell=m+t,(3.29)$ equals

$$
c\left(q_{m}(x), 4 m-\ell-2\right)+c\left(q_{m}(x), \ell-2\right)
$$

where $q_{m}(x)$ is as in 3.31 , and so (3.28) follows from 3.31, which is proved similarly to Lemma 3.30.

The proof when $k=2^{e+1}$ is similar and easier. It is also less important, since we don't need the result in this case, and hence is omitted.

Lemma 3.30. Let

$$
f_{m}(x)=\frac{\left(1+x^{2}+x^{4}\right)^{m}}{1+x^{3}}=\sum \alpha_{m, i} x^{i} .
$$

Then $\alpha_{m, i}+\alpha_{m, 4 m-3-i}=1$ for all integers $i$.

Proof. The proof is by induction on $m$. It is true for $m=1$ since $f_{1}(x)=$ $1+\sum_{i \geqslant 2} x^{i}$. Assume true for $m-1$. Note that $f_{m}(x)=\left(1+x^{2}+x^{4}\right) f_{m-1}(x)$. Thus

$$
\begin{aligned}
& \alpha_{m, i}+\alpha_{m, 4 m-3-i} \\
= & \alpha_{m-1, i}+\alpha_{m-1, i-2}+\alpha_{m-1, i-4}+\alpha_{m-1,4 m-3-i}+\alpha_{m-1,4 m-5-i}+\alpha_{m-1,4 m-7-i} \\
= & 1+1+1=1 .
\end{aligned}
$$

Lemma 3.31. Let

$$
q_{m}(x)=\frac{\left(1+x^{2}+x^{4}\right)^{m}\left(1+x+x^{2}\right)}{1+x^{6}}=\sum \beta_{m, i} x^{i}
$$

with $m \geqslant 1$. Then $\beta_{m, 4 m-\ell-2}+\beta_{m, \ell-2}=\delta_{\ell}$, where $\delta_{\ell}= \begin{cases}1 & \ell \text { odd } \\ 0 & \ell \text { even. }\end{cases}$ 
The following result completes the proof of Theorem 1.3.

Theorem 3.32. The exceptional Lie group $E_{6}$ does not satisfy the Technical Hypothesis.

Proof. We use the order of the generators in the computer package LiE $([\mathbf{1 9}])$. Information about the types of the representations can be found in [17], although a different ordering of the generators is used there. Then $R\left(E_{6}\right)$ has basic representations $\rho_{1}, \ldots, \rho_{6}$ of dimension $27,78,351,2925,351$, and 27 , respectively, such that $\rho_{2}$ and $\rho_{4}$ are real, while $t\left(\rho_{1}\right)=\rho_{6}$ and $t\left(\rho_{3}\right)=\rho_{5}$. Thus $V:=\operatorname{Ind}_{\mathbf{R}}\left(H\left(E_{6}\right)\right)$ is a $\mathbf{Z} / 2$-vector space with basis $\left\{\widetilde{\rho}_{2}, \widetilde{\rho}_{4}, \widetilde{\rho}_{1} \widetilde{\rho}_{6}, \widetilde{\rho}_{3} \widetilde{\rho}_{5}\right\}$. We will show that no subspace complementary to $\left\langle\widetilde{\rho}_{1} \widetilde{\rho}_{6}, \widetilde{\rho}_{3} \widetilde{\rho}_{5}\right\rangle$ is a $\psi^{3}$-submodule.

By Lemma 3.33(ii), a complementary subspace which is a $\psi^{3}$-submodule must have basis

$$
\left\{v_{2}=\widetilde{\rho}_{2}+a \widetilde{\rho}_{1} \widetilde{\rho}_{6}+b \widetilde{\rho}_{3} \widetilde{\rho}_{5}, v_{4}=\widetilde{\rho}_{4}+c \widetilde{\rho}_{1} \widetilde{\rho}_{6}+d \widetilde{\rho}_{3} \widetilde{\rho}_{5}\right\}
$$

satisfying $\psi^{3}\left(v_{2}\right)=v_{4}$ and $\psi^{3}\left(v_{4}\right)=v_{2}$. From Lemma 3.33(i), we have, in $V$, $\psi^{3}\left(\widetilde{\rho}_{1} \widetilde{\rho}_{6}\right)=\widetilde{\rho}_{3} \widetilde{\rho}_{5}$ and $\psi^{3}\left(\widetilde{\rho}_{3} \widetilde{\rho}_{5}\right)=\widetilde{\rho}_{1} \widetilde{\rho}_{6}$. Thus, using 3.33 again,

$$
\begin{aligned}
& \psi^{3}\left(v_{2}\right)-v_{4}=(a-d+1) \widetilde{\rho}_{3} \widetilde{\rho}_{5}+(b-c+1) \widetilde{\rho}_{1} \widetilde{\rho}_{6} \\
& \psi^{3}\left(v_{4}\right)-v_{2}=(c-b) \widetilde{\rho}_{3} \widetilde{\rho}_{5}+(d-a) \widetilde{\rho}_{1} \widetilde{\rho}_{6} .
\end{aligned}
$$

It is clearly impossible to choose the scalars so that both of these are 0 .

Lemma 3.33. $i$. $\operatorname{Mod}\left(2, I^{2}\right)$,

$$
\begin{aligned}
& \psi^{3}\left(\widetilde{\rho}_{1}\right)=\widetilde{\rho}_{3}+\widetilde{\rho}_{4} \\
& \psi^{3}\left(\widetilde{\rho}_{3}\right)=\widetilde{\rho}_{3}+\widetilde{\rho}_{4}+\widetilde{\rho}_{5}+\widetilde{\rho}_{6} \\
& \psi^{3}\left(\widetilde{\rho}_{5}\right)=\widetilde{\rho}_{1}+\widetilde{\rho}_{3}+\widetilde{\rho}_{4}+\widetilde{\rho}_{5} \\
& \psi^{3}\left(\widetilde{\rho}_{6}\right)=\widetilde{\rho}_{4}+\widetilde{\rho}_{5} .
\end{aligned}
$$

ii. In $V$,

$$
\begin{aligned}
& \psi^{3}\left(\widetilde{\rho}_{2}\right)=\widetilde{\rho}_{4}+\widetilde{\rho}_{1} \widetilde{\rho}_{6}+\widetilde{\rho}_{3} \widetilde{\rho}_{5} \\
& \psi^{3}\left(\widetilde{\rho}_{4}\right)=\widetilde{\rho}_{2} .
\end{aligned}
$$

Proof. Part (i) can be proved by the LiE methods used in the proof of (ii). It can also be obtained from $[\mathbf{1 2}, 3.9]$ by conjugating the matrix of $\psi^{3}$ given there by the change-of-basis matrix given there. The $B_{i}$ in $[\mathbf{1 2}, 3.9]$ correspond to $\widetilde{\rho}_{i}$. Indeed the matrix of $\psi^{3}$ on the basis $\left\{\widetilde{\rho}_{1}, \ldots, \widetilde{\rho}_{6}\right\}$ of $I / I^{2}$ is

$$
\left(\begin{array}{cccccc}
378 & -2079 & -143856 & 6062445 & -75843 & 0 \\
0 & 2430 & 46656 & -3109185 & 46656 & 0 \\
-27 & 351 & 18225 & -873261 & 11961 & 0 \\
1 & -77 & -2405 & 146586 & -2405 & 1 \\
0 & 351 & 11961 & -873261 & 18225 & -27 \\
0 & -2079 & -75843 & 6062445 & -143856 & 378
\end{array}\right)
$$

The proof of part (ii) requires LiE, and an algorithm somewhat similar to that used in [12]. Irreducible representations are represented in LiE by their highest 
weight, which, for $E_{6}$, is a 6 -tuple of integers. We adopt a notation that $\rho_{i_{1}, \ldots, i_{r}}$ with $1 \leqslant i_{1} \leqslant \cdots \leqslant i_{r} \leqslant 6$ has highest weight $\left(e_{1}, \ldots, e_{6}\right)$ where $e_{j}$ equals the number of $k$ for which $i_{k}=j$. For example, $\rho_{1,6}$ has highest weight $(1,0,0,0,0,1)$ and $\rho_{4,4}$ has highest weight $(0,0,0,2,0,0)$. The following information is obtained from LiE.

$$
\begin{aligned}
\rho_{1} \rho_{6} & =\rho_{1,6}+\rho_{2}+1 \\
\rho_{1} \rho_{2} & =\rho_{1,2}+\rho_{1}+\rho_{5} \\
\rho_{1} \rho_{3} & =\rho_{1,3}+\rho_{1,6}+\rho_{2}+\rho_{4} \\
\rho_{2}^{2} & =\rho_{2,2}+\rho_{1,6}+\rho_{2}+\rho_{4}+1 \\
\rho_{5} \rho_{6} & =\rho_{5,6}+\rho_{1,6}+\rho_{2}+\rho_{4} \\
\rho_{1,2} \rho_{6} & =\rho_{1,2,6}+\rho_{1,3}+\rho_{1,6}+\rho_{2,2}+\rho_{2}+\rho_{4} \\
\rho_{3} \rho_{5} & =\rho_{3,5}+\rho_{1,2,6}+\rho_{1,3}+\rho_{2,2}+2 \rho_{1,6}+\rho_{2}+\rho_{4}+\rho_{5,6}+1 \\
\rho_{2} \rho_{4} & =\rho_{2,4}+\rho_{1,2,6}+\rho_{1,3}+\rho_{1,6}+\rho_{2,2}+\rho_{2}+\rho_{3,5}+\rho_{4}+\rho_{5,6} \\
\rho_{2} \rho_{2,2} & =\rho_{2,2,2}+\rho_{1,2,6}+\rho_{2,2}+\rho_{2,4}+\rho_{2}+\rho_{4} \\
\psi^{3}\left(\rho_{2}\right) & =1+\rho_{5,6}+\rho_{4}+\rho_{3,5}+\rho_{2}+\rho_{2,4}+\rho_{2,2,2}+\rho_{1,6}+\rho_{1,3}
\end{aligned}
$$

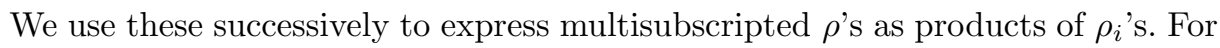
example, the first one yields

$$
\rho_{1,6}=\rho_{1} \rho_{6}-\rho_{2}-1
$$

and the third then yields

$$
\rho_{1,3}=\rho_{1} \rho_{3}-\rho_{2}-\rho_{4}-\left(\rho_{1} \rho_{6}-\rho_{2}-1\right) .
$$

Ultimately we obtain, mod 2 ,

$$
\psi^{3}\left(\rho_{2}\right) \equiv 1+\rho_{1} \rho_{6}+\rho_{1} \rho_{2} \rho_{6}+\rho_{3} \rho_{5}+\rho_{2} \rho_{4}+\rho_{4}+\rho_{2}^{3} .
$$

We have, $\bmod 2, \widetilde{\rho}_{2} \equiv \rho_{2}$, while if $i \neq 2$, then $\widetilde{\rho}_{i} \equiv \rho_{i}+1$. Substituting these into (3.34) yields the first equation of part (ii).

The second equation of part (ii) is proved by the same algorithm, but there are so many terms that it is only feasible to have a computer program manage all the details. We list the LiE program for completeness. The program just computes the coefficients of $1, \widetilde{\rho}_{1}, \widetilde{\rho}_{3}, \widetilde{\rho}_{5}, \widetilde{\rho}_{6}, \widetilde{\rho}_{1} \widetilde{\rho}_{6}$, and $\widetilde{\rho}_{3} \widetilde{\rho}_{5}$ in $\psi^{3}\left(\rho_{i}\right)$. The first five coefficients are needed in the determination of the last two. They also serve as a check on the validity of our program. We obtain that, mod 2 ,

$$
\psi^{3}\left(\rho_{4}\right)=1+\widetilde{\rho}_{1}+\widetilde{\rho}_{3}+\widetilde{\rho}_{5}+\widetilde{\rho}_{6}+0 \widetilde{\rho}_{1} \widetilde{\rho}_{6}+0 \widetilde{\rho}_{3} \widetilde{\rho}_{5}
$$

plus other terms. The last two coefficients of (3.35) yield the quadratic part of part (ii). (The linear part is contained in the matrix above.)

Note that coefficients 2 to 5 of (3.35) agree with those of the matrix above. (The first coefficient equals $\operatorname{dim}\left(\rho_{4}\right) \bmod 2$. Note also that (3.35) is not concerned with the coefficient of $\widetilde{\rho}_{2}$.) An additional check of our program was provided by modifying it to compute the same seven coefficients in $\psi^{3}\left(\rho_{2}\right)$. The values obtained, $0+0 \widetilde{\rho}_{1}+\widetilde{\rho}_{3}+\widetilde{\rho}_{5}+\widetilde{\rho}_{6}+\widetilde{\rho}_{1} \widetilde{\rho}_{6}+\widetilde{\rho}_{3} \widetilde{\rho}_{5}$, agreed with results obtained independently above. 
The LiE program is listed below. The reader may profit by comparing with the related program in $[\mathbf{1 2}, \S 7]$. Row numbers here are not part of the program; they are here for purpose of reference.

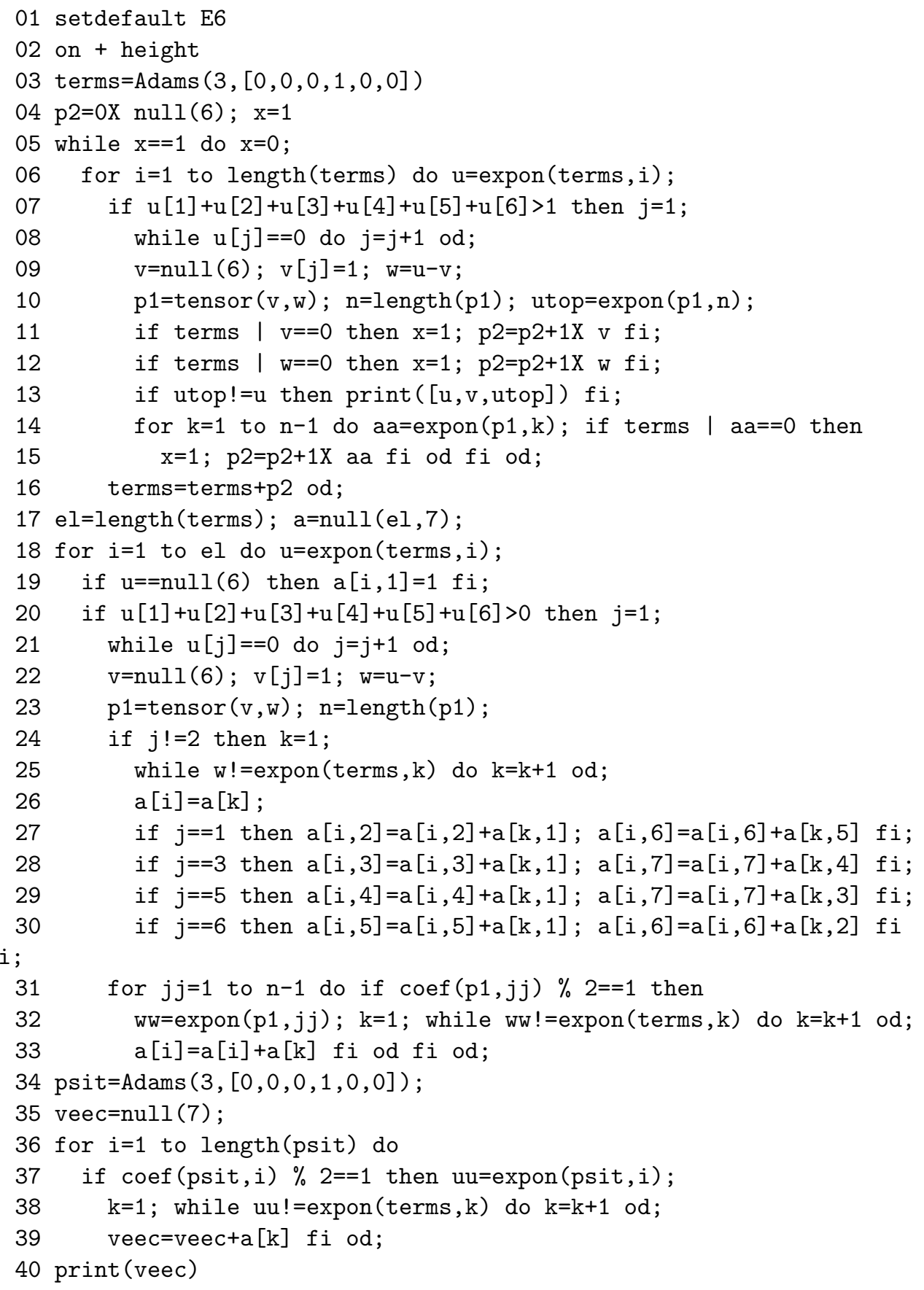


For each $\rho_{j_{1}, \ldots, j_{r}}$ that appears in $\psi^{3}\left(\rho_{i}\right)$, we decompose it as $\rho_{j_{1}} \otimes \rho_{j_{2}, \ldots, j_{r}}$ minus lower terms. The first loop adds to a list of terms all those lower terms not already present in the list (which starts with the terms of $\psi^{3}\left(\rho_{i}\right)$ ). This process is iterated until it stabilizes (no more terms being added to the list).

The terms in the list are ordered by height. The first term in the list will be $\rho_{\emptyset}=1$, which has coefficients $a_{1}=1$ and $a_{j}=0$ for $j>1$ (line 19). For each $\rho_{J}=\rho_{j_{1}, \ldots, j_{r}}$ in the list, the loop from lines 18 to 33 computes the coefficients $a_{i}$ such that

$$
\rho_{J}=a_{1}+a_{2} \widetilde{\rho}_{1}+a_{3} \widetilde{\rho}_{3}+a_{4} \widetilde{\rho}_{5}+a_{5} \widetilde{\rho}_{6}+a_{6} \widetilde{\rho}_{1} \widetilde{\rho}_{6}+a_{7} \widetilde{\rho}_{3} \widetilde{\rho}_{5}
$$

plus other terms. For a term $\rho_{J}$ in the list, let $J^{\prime}=\left(j_{2}, \ldots, j_{r}\right)$. Write

$$
\rho_{j_{1}} \otimes \rho_{J^{\prime}}=\rho_{J}+\sum_{K<J} \alpha_{K} \rho_{K}, \alpha_{K} \in \mathbf{Z} .
$$

The vector of coefficients of $\rho_{J}$ will include the sum of the vectors of those $\rho_{K}$ for which $\alpha_{K}$ is odd (lines 31 to 33 ).

Noting that $\rho_{j_{1}}=\widetilde{\rho}_{j_{1}}+1$ if $j_{1} \neq 2$, while $\rho_{2}=\widetilde{\rho}_{2}$, the $\rho_{j_{1}} \otimes \rho_{J^{\prime}}$ itself contributes nothing if $j_{1}=2$. Otherwise, it finds $\rho_{J^{\prime}}$ earlier in the list (line 25) and contributes the vector of coefficients of $\rho_{J^{\prime}}$ (due to the 1 in $\rho_{j_{1}}$ ) (line 26) plus, if $j_{1}=1,3,5$, or 6 , appropriate terms. For example, if $j_{1}=1$, the $\widetilde{\rho}_{1}$-term is increased by the coefficient of the constant term of $\rho_{J^{\prime}}$, and the $\widetilde{\rho}_{1} \widetilde{\rho}_{6}$-term is increased by the coefficient of $\widetilde{\rho}_{6}$ in $\rho_{J^{\prime}}$ (line 27). Finally, in lines 36 to 39 , we add the coefficient sequences of those $\rho_{J}$ which appear with odd coefficient in $\psi^{3}\left(\rho_{i}\right)$.

\section{4. $v_{1}$-periodic homotopy groups of $E_{8}$}

In this section, we prove Theorem 1.2 , the determination of the groups $v_{1}^{-1} \pi_{*}\left(E_{8} ; 2\right)$. The situation for $E_{8}$ is, at least conceptually, simpler than that for $E_{7}$ because all of its representations are real. The following proposition is an immediate consequence of Theorem 2.5, since the hypothesis implies $Q_{\mathbf{H}}=2 Q$.

Proposition 4.1. Let $G$ be a simply-connected compact Lie group, such as $G_{2}, F_{4}$, $E_{8}$, and $\operatorname{Spin}(n)$ with $n \equiv-1,0,1 \bmod 8$, which satisfies

- the Technical Condition 2.4,

- all representations are real, and

- $\lambda^{2}$ acts monomorphically on $Q$, where $Q$ is as in 2.2.

Let $K=\operatorname{ker}\left(\lambda^{2}: Q / 2 \rightarrow Q / 2\right)$ and $C=\operatorname{coker}\left(\lambda^{2}: Q / 2 \rightarrow Q / 2\right)$. Then

$$
K O^{i}\left(\Phi(G) ; \mathbf{Z}_{2}^{\wedge}\right) \approx \begin{cases}0 & i=0,1,2 \\ 2 Q / \lambda^{2}(2 Q) & i=3 \\ K & i=4 \\ C \# K & i=5 \\ C & i=6 \\ Q / \lambda^{2}(Q) & i=7 .\end{cases}
$$


The \# notation used here is as defined prior to Theorem 1.1.

The next result will be applied, with specific calculations, to give 1.2.

Proposition 4.2. Suppose $G$ is as in 4.1. Let $\theta_{m}=\lambda^{3}-3^{m}$ and $\bar{\theta}=\lambda^{3}-1$. Let

$$
\begin{aligned}
C_{m} & =\operatorname{cok}\left(\theta_{m} \mid Q / \operatorname{im}\left(\lambda^{2}\right)\right) \\
K_{m} & =\operatorname{ker}\left(\theta_{m} \mid Q / \operatorname{im}\left(\lambda^{2}\right)\right) \\
C K & =\operatorname{cok}\left(\bar{\theta} \mid \operatorname{ker}\left(\lambda^{2} \mid Q / 2\right)\right) \\
K K & =\operatorname{ker}\left(\bar{\theta} \mid \operatorname{ker}\left(\lambda^{2} \mid Q / 2\right)\right) \\
K C & =\operatorname{ker}\left(\bar{\theta} \mid \operatorname{cok}\left(\lambda^{2} \mid Q / 2\right)\right) \\
C C & =\operatorname{cok}\left(\bar{\theta} \mid \operatorname{cok}\left(\lambda^{2} \mid Q / 2\right)\right) .
\end{aligned}
$$

Then

$$
v_{1}^{-1} \pi_{8 k+d}(G)^{\#} \approx \begin{cases}K_{4 k-1} & d=-3 \\ C_{4 k-1} \oplus K K & d=-2 \\ (C K \oplus K C) \# K K & d=-1 \\ (C C \# K C) \oplus C K & d=0 \\ K_{4 k+1} \oplus C C & d=1 \\ C_{4 k+1} & d=2 \\ 0 & d=3,4 .\end{cases}
$$

Proof. For the most part, this result follows directly from 2.1 and 4.1. We must explain $\theta_{m}$ and the splittings.

The $\psi^{3}-9$ in $K O^{4 j-1}\left(\Phi G ; \mathbf{Z}_{2}^{\wedge}\right)$ which appears in 2.1 corresponds to $\psi^{3}-9$ in $K^{4 j-1}\left(\Phi G ; \mathbf{Z}_{2}^{\wedge}\right)$ under the realification and complexification homomorphisms, one of which is an isomorphism. Under Bott periodicity, this corresponds to $3^{1-2 j} \psi^{3}-9$ in $K^{1}\left(\Phi G ; \mathbf{Z}_{2}^{\wedge}\right)$, which is, by the proof of 2.5 , isomorphic to $P K^{1}\left(G ; \mathbf{Z}_{2}^{\wedge}\right) / \operatorname{im}\left(\psi^{2}\right)$, and this is isomorphic to $Q / \operatorname{im}\left(\lambda^{2}\right)$ with $\psi^{3}$ in $P K^{1}(G)$ corresponding to $\lambda^{3}$ in $Q$. Thus, $\psi^{3}-9$ in $K O^{4 j-1}(\Phi G)$ corresponds to $3^{1-2 j}\left(\lambda^{3}-3^{2 j+1}\right)$ in $Q$, as claimed in 4.2 , since this $\psi^{3}-9$ fits between $v_{1}^{-1} \pi_{4 j+1}(G)^{\#}$ and $v_{1}^{-1} \pi_{4 j+2}(G)^{\#}$.

The exact sequences of 2.1 and 2.2 lead to extension questions. That some of these are split can probably be derived from a more careful analysis of the work in [9] that led Bousfield to 2.2. We prefer to establish these split extensions by comparison with the Bendersky-Thompson spectral sequence (BTSS) method used in [3] and [4]. This comparison is quite illuminating for both approaches.

We employ the notation of 4.2 in a BTSS chart which generalizes $[\mathbf{3}, 4.9]$ for $G_{2}$ and $F_{4}$ and $[\mathbf{4}, 1.5]$ for $\operatorname{Spin}(8 a \pm 1)$. 
Diagram 4.3. The BTSS when all representations are real

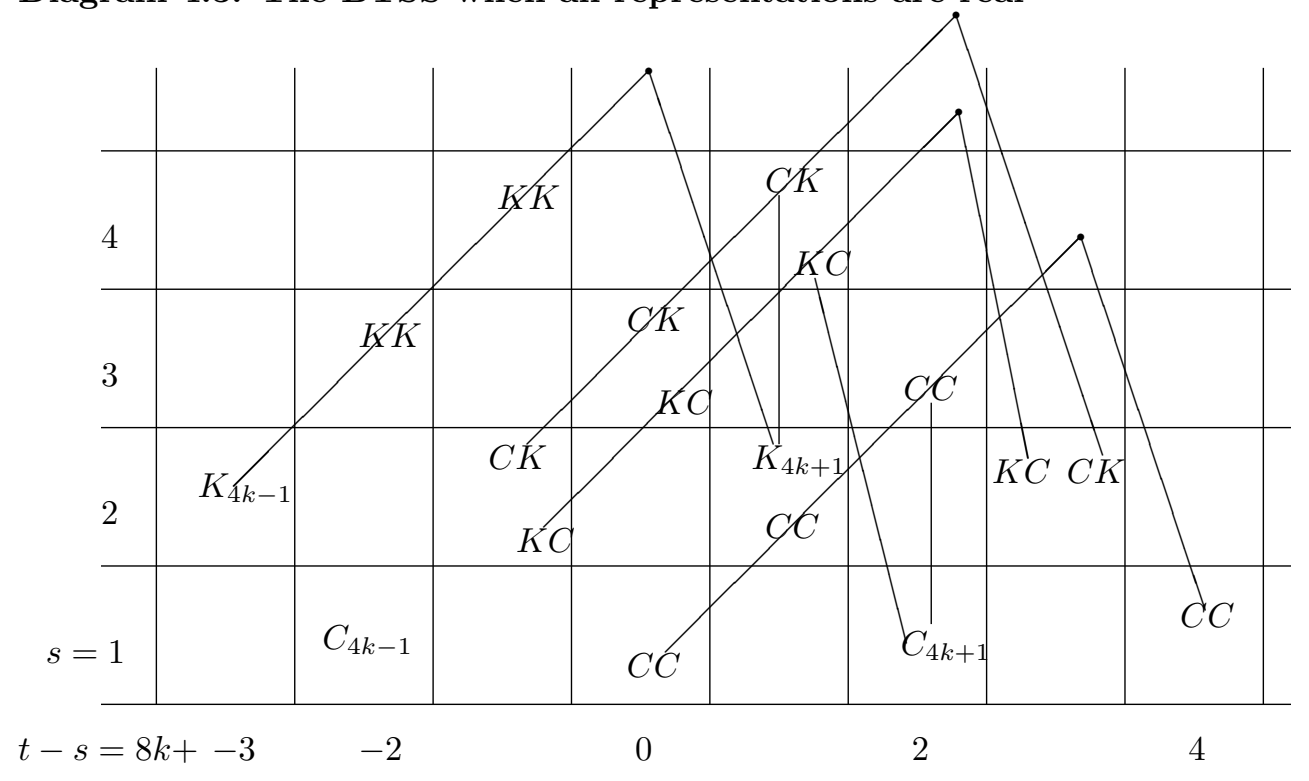

For $G_{2}, F_{4}$, and $\operatorname{Spin}(8 a \pm 1)$, the $d_{3}$-differentials were determined in $[\mathbf{3}]$ and [4] by naturality arguments. Such arguments seem to be inadequate to determine all $d_{3}$ 's in $E_{8}$. But 2.2, 4.1, and 4.2 imply that the differentials must behave as displayed above in all these spaces. The most subtle part is the differentials and extensions from $K_{4 k+1}$ and $C_{4 k+1}$. One way to see this is that the class(es) $K K$ in position $(8 k, 5)$ do not survive by 4.2 , and so must be hit by $d_{3}$ from $K_{4 k+1}$. But the $K_{4 k+1}$-summand in $v_{1}^{-1} \pi_{8 k+1}(G)$ is isomorphic to the summand in $E_{2}^{2,8 k+3}(G)$, and so the extension into $C K$ must be present to compensate for the class in $K_{4 k+1}$ which supported the $d_{3}$. A similar argument implies the differential and extension from $C_{4 k+1}$.

This chart then implies the $\oplus$ 's in 4.2. The one when $d=-2$ is due to the relation $2 \eta=0$ in $\pi_{*}(-)$. The $\oplus$ when $d=-1$ is due to the fact that $C K$ and $K C$ appear in the same filtration, and the direct sum splitting there was established in $[\mathbf{3}, 3.1]$. The splittings when $d=0$ and $d=1$ follow for similar reasons.

The reader should be aware that this BTSS comparison is only used to establish these splitting results, which should be regarded as fine tuning. Moreover, most of these splittings can probably be deduced from a deeper study of Bousfield's approach without the BTSS. A reader who is well-versed in such spectral sequences may draw additional insight from this comparison, but one who is not may omit this portion of the paper. That is the reason that we have not explained the spectral sequence in more detail.

Next we compute the groups of 4.2 explicitly for $E_{8}$. For Lie groups such as $E_{7}$, in which the division into type is nontrivial, it is important that we use the basis of $\widetilde{\rho}_{i}$ for our Adams operations. But in $E_{8}$ all representations are real, and so we may use any basis we like. Recall that $\left(Q, \lambda^{2}, \lambda^{3}\right)$ of 4.2 is isomorphic to $\left(P K^{1}(G),-\psi^{2}, \psi^{3}\right)$, which was computed in [12]. The main advantage of the $K$-theory perspective is 
that determination of $\psi^{2}$ is enough to obtain all $\psi^{k}$ because of the rational splitting of these Lie groups as products of spheres and the nice form of the Adams operations in spheres.

In $[\mathbf{1 2},(3.2)]$, a basis of eigenvectors of $\psi^{2}$ (and hence of all $\left.\psi^{k}\right)$ on $P K^{1}\left(E_{8} ; \mathbf{Q}\right)$ is given. These are expressed there in terms of $B_{i}=\beta\left(\widetilde{\rho}_{i}\right)$. These eigenvectors do not span integrally, and so in [12, pp.11-19], we describe an algorithm of replacing vectors $v$ by integral vectors $(v-w) / p$ to reduce the exponent of $p$ in $\operatorname{det}(M)$, where $M$ is the matrix having the vectors as columns. All Adams operations on all these vectors are explicitly known, since they are explicit combinations of eigenvectors. In [12], this procedure was implemented three times for $E_{8}$, once (3.5) to eliminate all powers of 5 from $\operatorname{det}(M)$, yielding $\psi^{k}$ on a basis of $P K^{1}\left(E_{8}\right)_{(5)}$, once (6.1) to eliminate all powers of 3 , and once (3.11) to eliminate all powers of all primes, yielding $\psi^{k}$ on a basis of $P K^{1}\left(E_{8}\right)$ (integral). Although we could use the latter here, the numbers are so large as to be unwieldy.

We perform the same algorithm to eliminate just the exponents of 2 . We start with the eight eigenvectors of $[\mathbf{1 2},(3.2)]$. At each stage, we find a combination of them which is even, and replace the first vector in that combination by the combination divided by 2 . Not only do we keep track of these vectors of integers, but also of the coefficients in the combinations. After 61 such steps, we have a set of eight vectors of integers such that $\operatorname{det}(M)$ is odd, and we have a matrix $N$ whose columns express the new vectors in terms of the original vectors. For example, if $\left\{v_{i}\right\}$ are the original vectors and $\left\{w_{i}\right\}$ the final ones, then

$$
w_{5}=\frac{1}{512} v_{5}-\frac{3}{512} v_{6}+\frac{7}{512} v_{7}-\frac{451}{512} v_{8},
$$

the coefficients of which appear in the second half of the fifth column of $N$. The matrix of $\psi^{k}$ with respect to this basis of $P K^{1}\left(E_{8}\right)_{(2)}$ equals $N D_{k} N^{-1}$, where $D_{k}$ is the diagonal matrix with entries

$$
\left(k, k^{7}, k^{11}, k^{13}, k^{17}, k^{19}, k^{23}, k^{29}\right) .
$$

We obtain the following matrices, which we write in transposed form, to fit them on one line.

$$
\left(\psi^{2}\right)^{T}=\left(\begin{array}{cccccccc}
2 & -4095 & 359169 & 516129 & 442353 & 76155 & 3045795 & -65866251 \\
0 & 128 & -20400 & 22242 & 2241 & -436758 & -1122003 & -154822161 \\
0 & 0 & 2048 & -17664 & -33408 & 321744 & -2942091 & -50472798 \\
0 & 0 & 0 & 8192 & -92160 & -69120 & -2018880 & 130940625 \\
0 & 0 & 0 & 0 & 2^{17} & -294912 & 626688 & -472681728 \\
0 & 0 & 0 & 0 & 0 & 2^{19} & -7372800 & 57741312 \\
0 & 0 & 0 & 0 & 0 & 0 & 2^{23} & -66060288 \\
0 & 0 & 0 & 0 & 0 & 0 & 0 & 2^{29},
\end{array}\right)
$$

while $\left(\psi^{3}\right)^{T}$ equals

$$
\begin{aligned}
& -70980 \text { equals } \\
& \begin{array}{ccccccc}
-70980 & 34109991 & -9760074 & -2500923 & -54666120 & 30971318970 & -8501480380389 \\
2187 & -1858950 & 10626633 & -3418281 & -1003550877 & -8902699632 & -19780047779454
\end{array} \\
& \begin{array}{ccccccc}
2187 & -1858950 & 10626633 & -3418281 & -1003550877 & -8902699632 & -19780047779454 \\
0 & 3^{11} & -4074381 & -23206257 & 754291926 & -35698131999 & -6402009498057
\end{array} \\
& \begin{array}{lcccccc}
0 & 0 & 3^{13} & -95659380 & -71744535 & -22121231625 & 16777754459505
\end{array}
\end{aligned}
$$

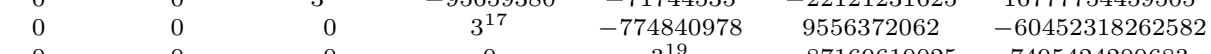

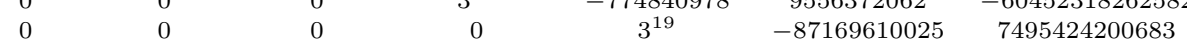

$$
\begin{aligned}
& \begin{array}{ccccccc}
0 & 0 & 0 & 0 & 0 & 3^{23} & -8567029273257 \\
0 & 0 & 0 & 0 & 0 & 0 & 3^{29}
\end{array}
\end{aligned}
$$
$\bar{\theta}$ of 4.2 sending $w_{5} \mapsto 0, w_{6} \mapsto w_{7}+w_{8}, w_{7} \mapsto w_{8}$, and $w_{8} \mapsto 0$. Thus in 4.2, 
$\operatorname{dim}(K K)=\operatorname{dim}(C K)=2$. Similarly $C$ of 4.1 is $\left\langle w_{1}, w_{2}, w_{3}, w_{4}, w_{6}: w_{2}+w_{3}+\right.$ $\left.w_{4}+w_{6}\right\rangle$ with $w_{1} \mapsto w_{3}, w_{2} \mapsto w_{4}+w_{6}, w_{3} \mapsto w_{4}, w_{4} \mapsto w_{6}$, and $w_{6} \mapsto 0$. Hence $\operatorname{dim}(K C)=\operatorname{dim}(C C)=1$.

We find the groups $C_{4 k \pm 1}$ of 4.2 by using the algorithm which was applied to $\left(E_{8}, 3\right)$ in $\left[\mathbf{1 2}\right.$, pp.35-37], to $\left(F_{4}, 2\right)$ in $[\mathbf{3}, 4.3]$, and to $\left(E_{6}, 2\right)$ in $[\mathbf{1 4}, 2.4]$. For $C_{m}$, we use Maple to pivot $\left(\begin{array}{c}\left(\psi^{2}\right)^{T} \\ \left(\psi^{3}-3^{m}\right)^{T}\end{array}\right)$ on odd entries and remove the corresponding row and column. This can be done seven times, leaving a $9 \times 1$ matrix, corresponding to the first column of the original matrix. Thus the group presented is cyclic of order $2^{e}$, with $e$ the smallest 2-exponent of the nine relations.

If $m \equiv 1 \bmod 8$, we eventually find it convenient to let $R=3^{m}-3^{2^{11}+273}$; that is, we consider $\left(\psi^{3}-R-3^{2^{11}+273}\right)^{T}$. Then $\nu(R)=\nu\left(m-2^{11}-273\right)+2 \geqslant 5$. We can reduce $3^{2^{11}+273} \bmod$ a suitable power of 2 , such as $2^{28}$, to keep the numbers manageable. The nine relations are expressed as polynomials in $R$. Two of them, corresponding to the 15 th and $14 \mathrm{th}^{1}$ rows of the original matrix, have lower 2 -powers than the others. Up to odd multiples, these are

$$
\begin{aligned}
& 2^{25}+2^{14} R+2^{10} R^{2}+2^{8} R^{3}+2^{4} R^{4}+2^{5} R^{5} \\
& 2^{24}+2^{10} R+2^{6} R^{2}+2^{4} R^{3}+R^{4}+2 R^{5} .
\end{aligned}
$$

The minimal 2-exponent is thus $\min \left(25, \nu\left(R-2^{14}\right)+10\right)$, which yields the first case of $e(m)$ in 1.2 .

The same two rows provide the minimal 2-exponents $e$ in the other congruences of $m$. They are

- If $m \equiv 3 \bmod 8$, let $R=3^{m}-3^{2^{14}+2^{11}+19}$. Then $e=\min \left(28, \nu\left(R-2^{17}\right)+10\right)$, due to relations

$$
\begin{aligned}
& 2^{28}+2^{14} R+2^{10} R^{2}+2^{8} R^{3}+2^{4} R^{4}+2^{5} R^{5} \\
& 2^{27}+2^{10} R+2^{6} R^{2}+2^{4} R^{3}+R^{4}+2 R^{5} .
\end{aligned}
$$

- If $m \equiv 5 \bmod 8$, let $R=3^{m}-3^{2^{25}+2^{23}+2^{22}+2^{20}+29}$. Then $e=\min (39, \nu(R)+$ 10) due to relations

$$
\begin{aligned}
& 2^{39}+2^{14} R+2^{10} R^{2}+2^{8} R^{3}+2^{4} R^{4}+2^{5} R^{5} \\
& 2^{40}+2^{10} R+2^{6} R^{2}+2^{4} R^{3}+R^{4}+2 R^{5} .
\end{aligned}
$$

- If $m \equiv 7 \bmod 8$, let $R=3^{m}-3^{2^{17}+23}$. Then $e=\min (31, \nu(R)+10)$ due to relations

$$
\begin{aligned}
& 2^{31}+2^{14} R+2^{10} R^{2}+2^{8} R^{3}+2^{4} R^{4}+2^{5} R^{5} \\
& 2^{33}+2^{10} R+2^{6} R^{2}+2^{4} R^{3}+R^{4}+2 R^{5} .
\end{aligned}
$$

This shows that the groups $C_{m}$ are as claimed in 1.2. For the groups $K_{m}$, we use Proposition 4.4 to see that $K_{m}$ is isomorphic to the group presented by $\left(\begin{array}{c}\psi^{2} \\ \psi^{3}-3^{m}\end{array}\right)$; i.e., the two component matrices are no longer in transposed form. When pivoting is performed on this matrix, after six steps of pivoting on an odd element, all remaining

${ }^{1}$ The 12 th row gives the same exponents as the 14 th row. 
elements are even. One of them has $\nu(-)=1$. Since $K_{m}$ has the same order as $C_{m}$, we obtain $K_{m} \approx \mathbf{Z} / 2^{e(m)-1} \oplus \mathbf{Z}_{2}$. This completes the proof of Theorem 1.2.

The following proposition, which was used above, is a slight generalization of a portion of $[\mathbf{4}, 11.3]$.

Proposition 4.4. Let $G$ be a finitely generated free abelian group, and $\theta$ and $\psi$ commuting injective endomorphisms of $G$ with finite cokernels. Let

$$
K=\operatorname{ker}(G / \operatorname{im}(\psi) \stackrel{\theta}{\longrightarrow} G / \operatorname{im}(\psi)) .
$$

Then $K^{\#}$ is presented by $\left(\begin{array}{c}(\psi) \\ (\theta)\end{array}\right)$, where $(\psi)$ and $(\theta)$ denote matrices of $\psi$ and $\theta$ with respect to any fixed basis.

Proof. Since Pontrjagin duality is exact, the sequence

$$
(G / \operatorname{im}(\psi))^{\#} \stackrel{\theta^{*}}{\longrightarrow}(G / \operatorname{im}(\psi))^{\#} \rightarrow K^{\#} \rightarrow 0
$$

is exact. If $F$ is a finite abelian group, there is a natural isomorphism $F^{\#} \rightarrow$ $\operatorname{Ext}(F, \mathbf{Z})$ induced from the short exact sequence $0 \rightarrow \mathbf{Z} \rightarrow \mathbf{Q} \rightarrow \mathbf{Q} / \mathbf{Z} \rightarrow 0$. Also, there is an isomorphism

$$
\operatorname{Ext}(G / \operatorname{im}(\psi), \mathbf{Z}) \approx \operatorname{Hom}(G, \mathbf{Z}) / \operatorname{im}\left(\psi^{*}\right) .
$$

Thus

$$
K^{\#} \approx \operatorname{Hom}(G, \mathbf{Z}) / \operatorname{im}\left(\psi^{*}, \theta^{*}\right) .
$$

On the basis of $\operatorname{Hom}(G, \mathbf{Z})$ dual to that of $G$, the matrices of $\psi^{*}$ and $\theta^{*}$ are $(\psi)^{T}$ and $(\theta)^{T}$, but when we take the presentation matrix as in (4.5), we transpose back again.

\section{5. $v_{1}$-periodic homotopy groups of $E_{7}$}

Although its numbers are not quite as large, the computation of $v_{1}^{-1} \pi_{*}\left(E_{7}\right)$ is slightly more complicated than that of $v_{1}^{-1} \pi_{*}\left(E_{8}\right)$ because not all representations are real. We must work with the basis $\widetilde{\rho}_{1}, \ldots, \widetilde{\rho}_{7}$ of $I / I^{2}$ or its corresponding basis $B_{1}, \ldots, B_{7}$ of $P K^{1}\left(E_{7}\right)$. Here $B_{i}=\beta\left(\widetilde{\rho}_{i}\right)$.

The ordering of the basic representations in LiE has $\rho_{1}, \ldots, \rho_{7}$ of dimensions 133 , $912,8645,365750,27664,1539$, and 56, respectively, with $\rho_{2}, \rho_{5}$, and $\rho_{7}$ quaternionic, and the rest real.

Proposition 5.1. With respect to the basis $\left\{B_{1}, \ldots, B_{7}\right\}$ of $P K^{1}\left(E_{7}\right)$, the matrices of $\psi^{2}$ and $\psi^{3}$ are as follows.

$$
\psi^{2}=\left(\begin{array}{ccccccc}
132 & -264 & -399664 & 186729664 & -1545576 & 0 & 0 \\
0 & 856 & 27664 & -28553504 & 276696 & 0 & 0 \\
-1 & 2 & 7372 & -3803952 & 33306 & 0 & 0 \\
0 & -1 & -133 & 110845 & -1273 & 1 & 0 \\
0 & 0 & 968 & -1275832 & 21184 & -56 & 0 \\
0 & 1 & -11857 & 18311345 & -350951 & 1541 & -1 \\
0 & -912 & 42560 & -127779712 & 3514352 & -27776 & 56
\end{array}\right)
$$




$$
\psi^{3}=\left(\begin{array}{ccccccc}
9043 & -1345048 & -239884976 & 182665852920 & -2039397624 & 1545576 & 0 \\
0 & 261306 & 32232816 & -27497867040 & 319559499 & -276696 & 0 \\
-133 & 27664 & 4775915 & -3712222926 & 41922720 & -33306 & 0 \\
1 & -856 & -128402 & 108783702 & -1294848 & 1329 & 0 \\
0 & 8511 & 1404576 & -1295487792 & 16666478 & -24264 & 1 \\
0 & -113736 & -19888326 & 19028389419 & -253946608 & 435708 & -56 \\
0 & 564108 & 133440192 & -142676642736 & 2109092684 & -5069808 & 1597
\end{array}\right)
$$

Proof. The first matrix is obtained using the method of [12]. In [12, pp.8-9] is described the algorithm for finding $\lambda^{2}$ on $\widetilde{\rho}_{1}, \ldots, \widetilde{\rho}_{7}$. This involves running the LiE program in $[\mathbf{1 2}, \S 7]$ which gives $\lambda^{2}$ on $\rho_{1}, \ldots, \rho_{7}$, and then subtracting $\operatorname{dim}\left(\rho_{i}\right)$ from diagonal elements. This matrix is then negated to give $\psi^{2}$ on $\left\{B_{1}, \ldots, B_{7}\right\}$ in $P K^{1}\left(E_{7}\right)$.

We find eigenvectors of this matrix $\left(\psi^{2}\right)$, and then, as described in Section 4, repeatedly replace vectors $v$ by integral vectors $(v-w) / 2$ until we have a spanning set of vectors which are explicit combinations of eigenvectors, and hence on which we know all $\psi^{k}$. The change of basis matrix is

$$
M=\left(\begin{array}{ccccccc}
-95186445 & 2454179 & -27954 & 9434 & -12456 & 1800 & 1672 \\
-3705699 & 87516 & 3654 & 1221 & -1119 & -75 & -252 \\
-156975 & 3856 & -90 & -131 & 195 & -30 & -34 \\
-474 & 11 & 0 & -1 & 0 & 0 & 1 \\
-22225 & 534 & 18 & 0 & -3 & 15 & -12 \\
-297427 & 5403 & 693 & 501 & -123 & -330 & 177 \\
517780118 & -12819489 & -53820 & -16146 & 5820 & 4920 & -1344
\end{array}\right)
$$

Thus the columns of $M$ are combinations of $B_{i}$ 's with respect to which all $\psi^{k}$ have a known triangular form, similar to $[\mathbf{1 2}, 3.10]$. One can check that $\operatorname{det}(M)=$ $3^{16} 5^{7} 7^{4} 11^{2} 13^{2} 17$, reflecting that it spans localized at 2 , but not at the other primes relevant to $E_{7}$. We find that $\psi^{3}$ with respect to the basis given by the columns of $M$ is given by the following matrix $P_{3}$.

$$
\left(\begin{array}{ccccccc}
3 & 0 & 0 & 0 & 0 & 0 & 0 \\
-9600 & 243 & 0 & 0 & 0 & 0 & 0 \\
2316 & -4617 & 2187 & 0 & 0 & 0 & 0 \\
28272 & 18711 & -34992 & 19683 & 0 & 0 & 0 \\
66963 & 78246 & -188082 & -157464 & 177147 & 0 & 0 \\
-520551 & 279207 & 1264086 & 98415 & -1771470 & 1594323 & 0 \\
52172712 & -55209114 & -14020857 & -92313270 & -20371905 & -15943230 & 3^{17}
\end{array}\right)
$$

The matrix of $\psi^{3}$ in the statement of this theorem is obtained as $M P_{3} M^{-1}$.

Now we embark on the application of 2.1 and 2.2, which holds for $E_{7}$ by 2.5 and 3.4, to $E_{7}$. By 3.1 and 2.3 ,

$$
\begin{aligned}
& Q_{\mathbf{R}}=\left\langle\widetilde{\rho}_{1}, \widetilde{\rho}_{3}, \widetilde{\rho}_{4}, \widetilde{\rho}_{6}, 2 \widetilde{\rho}_{2}, 2 \widetilde{\rho}_{5}, 2 \widetilde{\rho}_{7}\right\rangle \\
& Q_{\mathbf{H}}=\left\langle 2 \widetilde{\rho}_{1}, 2 \widetilde{\rho}_{3}, 2 \widetilde{\rho}_{4}, 2 \widetilde{\rho}_{6}, \widetilde{\rho}_{2}, \widetilde{\rho}_{5}, \widetilde{\rho}_{7}\right\rangle .
\end{aligned}
$$

Using 2.2 and the $\psi^{2}$ matrix of 5.1 , we obtain

Proposition 5.2. Let $\mathbf{B}=P K^{1}\left(E_{7}\right), \mathbf{B}_{\mathbf{R}}=\left\langle B_{1}, B_{3}, B_{4}, B_{6}\right\rangle$, and $\mathbf{B}_{\mathbf{H}}=\left\langle B_{2}, B_{5}, B_{7}\right\rangle$. 
Let $\mathbf{D}=\left\langle B_{2}-B_{3}, B_{3}-B_{4}, B_{4}-B_{5}, B_{5}-B_{6}\right\rangle$. There are isomorphisms

$$
K O^{i}\left(\Phi E_{7}\right) \approx \begin{cases}0 & i=0 \\ \left\langle B_{2}, B_{5}, B_{7}\right\rangle / 2 & i=1,2 \\ \left\langle 2 \mathbf{B}_{\mathbf{R}}, \mathbf{B}_{\mathbf{H}}\right\rangle / \psi^{2}(2 \mathbf{B}) & i=3 \\ \mathbf{D} / 2 & i=4 \\ \left\langle B_{1}\right\rangle / 2 \# \mathbf{D} / 2 & i=5 \\ \left\langle B_{1}\right\rangle / 2 & i=6 \\ \left\langle\mathbf{B}_{\mathbf{R}}, 2 \mathbf{B}_{\mathbf{H}}\right\rangle / \psi^{2}(\mathbf{B}) & i=7 .\end{cases}
$$

We substitute this proposition into 2.1, using the matrix $\left(\psi^{3}\right)$ of 5.1 and obtain the following result.

Proposition 5.3. Let $\theta_{m}=\psi^{3}-3^{m}$. The groups $v_{1}^{-1} \pi_{8 k+d}\left(E_{7}\right)^{\#}$ are isomorphic to

$$
\begin{cases}\mathbf{Z}_{2}\left(B_{2}+B_{5}\right) & d=3 \\ \mathbf{Z}_{2}\left(B_{7}\right) \# \mathbf{Z}_{2}\left(B_{2}+B_{5}\right) & d=4 \\ \mathbf{Z}_{2}\left(B_{7}\right) \# \operatorname{ker}\left(\theta_{4 k+3} \mid\left\langle 2 \mathbf{B}_{\mathbf{R}}, \mathbf{B}_{\mathbf{H}}\right\rangle / \psi^{2}(2 \mathbf{B})\right) & d=5 \\ \left\langle 2 \mathbf{B}_{\mathbf{R}}, \mathbf{B}_{\mathbf{H}}\right\rangle /\left(\psi^{2}(2 \mathbf{B}), \theta_{4 k+3}\left\langle 2 \mathbf{B}_{\mathbf{R}}, \mathbf{B}_{\mathbf{H}}\right\rangle\right) \#\left(\mathbf{Z}_{2}\left(B_{4}-B_{6}\right) \oplus \mathbf{Z}_{2}\left(B_{2}-B_{5}\right)\right) & d=6 \\ \left(\mathbf{Z}_{2}\left(B_{2}-B_{3}\right) \oplus \mathbf{Z}_{2}\left(B_{3}-B_{4}\right)\right) \#\left(\mathbf{Z}_{2}\left(B_{1}\right) \#\left(\mathbf{Z}_{2}\left(B_{4}-B_{6}\right) \oplus \mathbf{Z}_{2}\left(B_{2}-B_{5}\right)\right)\right) & d=7 \\ \left(\mathbf{Z}_{2}\left(B_{1}\right) \#\left(\mathbf{Z}_{2}\left(B_{2}-B_{3}\right) \oplus \mathbf{Z}_{2}\left(B_{3}-B_{4}\right)\right)\right) \# \mathbf{Z}_{2}\left(B_{1}\right) & d=8 \\ \mathbf{Z}_{2}\left(B_{1}\right) \# \operatorname{ker}\left(\theta_{4 k+5} \mid\left\langle\mathbf{B}_{\mathbf{R}}, 2 \mathbf{B}_{\mathbf{H}}\right\rangle / \psi^{2}(\mathbf{B})\right) & d=9 \\ \left\langle\mathbf{B}_{\mathbf{R}}, 2 \mathbf{B}_{\mathbf{H}}\right\rangle /\left(\psi^{2}(\mathbf{B}), \theta_{4 k+5}\left\langle\mathbf{B}_{\mathbf{R}}, 2 \mathbf{B}_{\mathbf{H}}\right\rangle\right) & d=10\end{cases}
$$

As we did with $E_{8}$, we can resolve some of the extension questions by comparing with the BTSS. The study of the extension questions is facilitated by first comparing the large summands of 5.3 with the $E_{2}$-term of the BTSS. As with $E_{8}$, the BTSS is only needed for resolving the extension questions and providing possible insight.

We first consider the group $\left\langle 2 \mathbf{B}_{\mathbf{R}}, \mathbf{B}_{\mathbf{H}}\right\rangle /\left(\psi^{2}(2 \mathbf{B}), \theta_{4 k+3}\left\langle 2 \mathbf{B}_{\mathbf{R}}, \mathbf{B}_{\mathbf{H}}\right\rangle\right)$ which occurs in 5.3 when $d=6$. The corresponding group $E_{2}^{1,8 k+7}\left(E_{7}\right)^{\#}$ has the same form, with the three 2's omitted. We compute this latter group first.

The desired group $E_{2}^{1,8 k+7}\left(E_{7}\right)^{\#}$ is presented by the matrix

$$
M=\left(\begin{array}{c}
\left(\psi^{2}\right)^{T} \\
\left(\psi^{3}-3^{4 k+3}\right)^{T}
\end{array}\right),
$$

where $\left(\psi^{2}\right)$ and $\left(\psi^{3}\right)$ are as in 5.1. Five times we pivot on odd elements, removing rows and columns, to leave a $9 \times 2$ matrix. The smallest $\nu(-)$ of remaining terms is $\nu=2$, and so we split off a $\mathbf{Z} / 4$ and pivot on that element. We let $R=3^{4 k+3}-3^{75}$, and so $\nu(R)=\nu(k-18)+4$. Of the eight relations remaining, those with the smallest 2-exponents are, with odd multiples omitted,

$$
\begin{gathered}
2^{16}+2^{7} R+2^{6} R^{2}+2 R^{3} \\
2^{17}+2^{7} R+2^{4} R^{2}+2 R^{3} .
\end{gathered}
$$


We obtain

$$
E_{2}^{1,8 k+7}\left(E_{7}\right) \approx \mathbf{Z} / 4 \oplus \mathbf{Z} / 2^{\min (16, \nu(k-18)+11)} .
$$

Note that when we express a finite group $G^{\#}$ as a sum of cyclic summands, we may write it as $G$, since the two are abstractly isomorphic.

The effect of the three 2's that occur in the first part of $v_{1}^{-1} \pi_{8 k+6}\left(E_{7}\right)^{\#}$ in 5.3 is to multiply by 2 rows $1-8,10,11$, and 13 of the matrix $M$ of the preceding paragraph, and then divide by 2 columns $1,3,4$, and 6 . The five odd entries on which we pivoted in the previous paragraph are in positions ${ }^{2}$ which were unaffected by the multiplying and dividing by 2 that took place here, and we pivot on them as before. The $\mathbf{Z} / 4$ is in position $(8,1)$ and also remains unchanged by the multiplying and dividing by 2 and is still a $\mathbf{Z} / 4$ after the five steps of pivoting. After pivoting on the $\mathbf{Z} / 4$, and with $R$ as before, the first relation of (5.4) is now twice as large, while the second is not changed. ${ }^{3}$ Thus we obtain that the first part of $v_{1}^{-1} \pi_{8 k+6}\left(E_{7}\right)^{\#}$ in 5.3 is $\mathbf{Z} / 4 \oplus \mathbf{Z} / 2^{\min (17, \nu(k-18)+11)}$.

A similar analysis applies to the group in 5.3 with $d=10$. We first look at the corresponding BTSS group $E_{2}^{1,8 k+11}\left(E_{7}\right)^{\#}$, which has the same form without the two 2's. The pivoting up to and including splitting off the $\mathbf{Z} / 4$ is the same as in the case $d=6$ just considered. If $k$ is even, we let $R=3^{4 k+5}-3^{141}$, and so $\nu(R)=\nu(k-34)+4$. The relations with smallest 2 -exponents are

$$
\begin{gathered}
2^{20}+2^{8} R+2^{4} R^{2}+2 R^{3} \\
2^{19}+2^{8} R+2^{4} R^{2}+2 R^{3} .
\end{gathered}
$$

If $k$ is odd, we let $R=3^{4 k+5}-3^{7 \cdot 2^{11}+17}$, and so $\nu(R)=\nu\left(k-7 \cdot 2^{9}-3\right)+4$. The relations with smallest 2 -exponent are

$$
\begin{gathered}
2^{24}+2^{8} R+2^{4} R^{2}+2 R^{3} \\
2^{24}+2^{10} R+2^{6} R^{2}+2^{3} R^{3} .
\end{gathered}
$$

Thus we obtain

$$
E_{2}^{1,8 k+11}\left(E_{7}\right) \approx \mathbf{Z} / 4 \oplus \begin{cases}\mathbf{Z} / 2^{\min (19, \nu(k-34)+12)} & k \text { even } \\ \mathbf{Z} / 2^{\min \left(24, \nu\left(k-7 \cdot 2^{9}-3\right)+12\right)} & k \text { odd }\end{cases}
$$

To obtain $v_{1}^{-1} \pi_{8 k+10}\left(E_{7}\right)^{\#}$, we multiply rows 9,12 , and 14 by 2 , and then divide columns 2,5 , and 7 by 2 . The pivoting through the $\mathbf{Z} / 4$ goes as before, as the pivoted-upon elements were unchanged. Both the relevant relations $((5.6)$ or $(5.7))$ are divided by 2 , as they are in original row 3 or 4 and column 7 . Thus $v_{1}^{-1} \pi_{8 k+10}\left(E_{7}\right)$ is obtained from (5.8) by decreasing the exponent of the large summand by 1 .

In order to determine the ker part of $v_{1}^{-1} \pi_{8 k+9}\left(E_{7}\right)^{\#}$ in 5.3 explicitly, we need the following slight modification of Proposition 4.4, which is proved by the same method.

${ }^{2}(1,3),(2,4),(7,6),(14,5)$, and $(12,2)$

${ }^{3}$ The first relation occurs three times (with different odd coefficients) and is due to original rows 3,4 , and 6 , while the second relation comes from original row 9 . The generator for these relations is that of column 7 . 
Proposition 5.9. Suppose $G, \psi$, and $\theta$ are as in 4.4. Suppose the basis of $G$ is partitioned as $B_{1} \coprod B_{2}$. Let $G^{\prime}$ be the subgroup of $G$ spanned by $B_{1}$ and $2 B_{2}$, and assume $\psi(G) \subset G^{\prime}$. Then $\theta$ induces $\theta^{\prime}: G^{\prime} / \psi(G) \rightarrow G^{\prime} / \psi(G)$. Let $K^{\prime}=\operatorname{ker}\left(\theta^{\prime}\right)$. Then $\left(K^{\prime}\right)^{\#}$ is presented by

$$
\left(\begin{array}{cc}
A_{1} & A_{2} \\
\frac{1}{2} A_{3} & \frac{1}{2} A_{4} \\
C_{1} & 2 C_{2} \\
\frac{1}{2} C_{3} & C_{4}
\end{array}\right)
$$

where $\left(\begin{array}{ll}A_{1} & A_{2} \\ A_{3} & A_{4}\end{array}\right)$ and $\left(\begin{array}{ll}C_{1} & C_{2} \\ C_{3} & C_{4}\end{array}\right)$ are the matrices of $\psi$ and $\theta$ with respect to $B_{1} \amalg B_{2}$.

When this is computed using $\psi=\psi^{2}$ and $\theta=\psi^{3}-3^{4 k+5}$ of 5.1 using the pivoting methods described above, we obtain that the ker part of $v_{1}^{-1} \pi_{8 k+9}\left(E_{7}\right)^{\#}$ is $\mathbf{Z} / 2^{4} \oplus \mathbf{Z} / 2^{e-2}$, where $e$ is the 2-exponent of the large summand of $v_{1}^{-1} \pi_{8 k+10}\left(E_{7}\right)$ described above. Note that the two groups must have the same order since they are the kernel and cokernel of an endomorphism of a finite abelian group.

A result similar to 5.9 applies to the large summand of $v_{1}^{-1} \pi_{8 k+5}\left(E_{7}\right)$, and when it is computed we obtain similarly that the ker part of $v_{1}^{-1} \pi_{8 k+5}\left(E_{7}\right)^{\#}$ is $\mathbf{Z} / 2^{4} \oplus$ $\mathbf{Z} / 2^{e-2}$, where $e=\min (17, \nu(k-18)+11)$, the large summand in $v_{1}^{-1} \pi_{8 k+6}\left(E_{7}\right)$ obtained earlier. We emphasize that in performing the pivoting to obtain this group, we need find only that it has two summands and that the smaller has order $2^{4}$.

As we shall use the BTSS to settle some extension questions, it is useful to know the following.

Proposition 5.10. The BTSS 2-line groups are given by

$$
E_{2}^{2,8 k+d+2}\left(E_{7}\right) \approx \mathbf{Z}_{2} \oplus \begin{cases}\mathbf{Z} / 2^{4} \oplus \mathbf{Z} / 2^{t_{1}-2} & d=5 \\ \mathbf{Z} / 2^{5} \oplus \mathbf{Z} / 2^{t_{2}-3} & d=9,\end{cases}
$$

where $t_{1}$ is the large exponent in (5.5) and $t_{2}$ the large exponent in (5.8).

Proof. As in $[\mathbf{3}, 3.1 \mathrm{~b}]$, there is a short exact sequence

$$
0 \rightarrow \mathbf{B} /\left(2, \psi^{2}, \theta_{4 k+(d+1) / 2}\right) \rightarrow E_{2}^{2,8 k+d+2}\left(E_{7}\right)^{\#} \rightarrow \operatorname{ker}\left(\theta_{4 k+(d+1) / 2} \mid \mathbf{B} / \operatorname{im}\left(\psi^{2}\right)\right) \rightarrow 0 .
$$

The first group is $\mathbf{Z}_{2}$ generated by $B_{1}(d=9)$ or $B_{7}(d=5)$, while the second is obtained by the algorithm used to find $K_{m}$ for $E_{8}$ near the end of Section 4 or that used to find the groups $v_{1}^{-1} \pi_{8 k+d}\left(E_{7}\right)$ above, without the 2 's that complicated the argument there. The matrix pivots down to two columns, and the smallest 2exponent is $4(d=5)$ or $5(d=9)$. The other exponent is then forced by the equality of the orders of the kernel and cokernel of an endomorphism.

We can incorporate the above information into the BTSS chart for $E_{7}$ below, and then use this chart, as we did with $E_{8}$, to draw inferences about some extensions in 5.3. The facts that we use to make these inferences are that $2 \eta=0$ in $\pi_{*}(-)$ and that relationships between elements in the same bidegree is the same in $\pi_{*}(-)$ as it is in $E_{2}(-)$. Theorem 1.1 follows from 5.3, the specific calculations of portions of $v_{1}^{-1} \pi_{8 k+d}\left(E_{7}\right)$ made above when $d=5,6,9$, and 10 , and the extension inferences 
obtained from the chart. The dotted differential and extension take place unless $\nu(k-18) \geqslant 6$, in which case the exponent of the homotopy group is 1 larger than the $E_{2}$-exponent.

\section{Diagram 5.11. The BTSS of $E_{7}$}

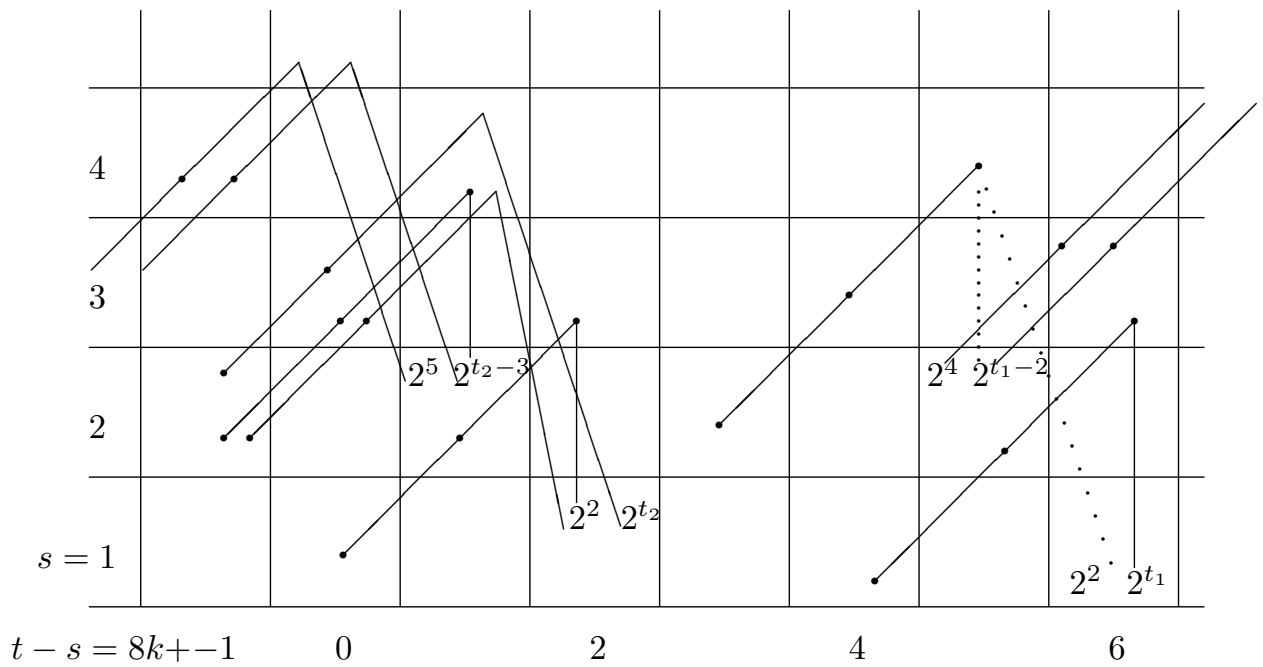

\section{References}

[1] M. Bendersky and D. M. Davis, 2-primary v-periodic homotopy groups of $S U(n)$, Amer Jour Math 114 (1991) 529-544.

[2] The 1-line of the K-theory Bousfield-Kan spectral sequence for Spin(2n+1), Contemp Math AMS, 279 (2001) 37-56.

[3] - A stable approach to an unstable homotopy spectral sequence, Topology 42 (2003) 1261-1287.

[4] $\longrightarrow$, v $v_{1}$-periodic homotopy groups of $S O(n)$, to appear in Memoirs Amer Math Soc.

[5] 3-primary $v_{1}$-periodic homotopy groups of $F_{4}$ and $E_{6}$, Trans Amer Math Soc 344 (1994) 291-306.

[6] M. Bendersky, D. M. Davis, and M. Mahowald, $v_{1}$-periodic homotopy groups of $S p(n)$, Pac Jour Math 170 (1995) 319-378.

[7] M. Bendersky, D. M. Davis, and M. Mimura, $v_{1}$-periodic homotopy groups of exceptional Lie groups - torsion-free cases, Trans Amer Math Soc 333 (1992) 115-136.

[8] A. K. Bousfield, The $K$-theory localization and $v_{1}$-periodic homotopy groups of H-spaces, Topology 38 (1999) 1239-1264. 
[9] A classification of K-local spectra, Jour Pure Appl Alg 66 (1990) 121-163.

[10] M. R. Bremner, R. V. Moody, and J. Patera, Tables of dominant weight multiplicities for representations of simple Lie algebras, Marcel Dekker (1985).

[11] T. Bröcker and T. tom Dieck, Representations of compact Lie groups, Springer-Verlag (1985).

[12] D. M. Davis, From representation theory to homotopy groups, Mem Amer Math Soc 759 (2002).

[13] $v_{1}$-periodic homotopy groups of $S U(n)$ at odd primes, Proc London Math Soc 43 (1991) 529-541.

[14] The K-completion of $E_{6}$, Progress in Mathematics 215 (2003) 107-124, Birkhauser.

[15] 3-primary $v_{1}$-periodic homotopy groups of $E_{7}$, New York Jour Math 4 (1998) 185-221.

[16] L. Hodgkin, On the K-theory of Lie groups, Topology 6 (1967) 1-36.

[17] W. G. McKay and J. Patera, Tables of dimensions, indices, and branching rules for representations of simple Lie algebras, Marcel Dekker (1981).

[18] C. M. Naylor, Cohomology operations in the K-theory of the classical groups, Port Math 38 (1979) 145-153.

[19] M. vanLeeuven, LiE, a computer algebra package for Lie algebra computations, http://wwwmathlabo.univ-poitiers.fr/ maavl/LiE/.

This article may be accessed via WWW at http://www.rmi.acnet.ge/hha/ or by anonymous ftp at

ftp://ftp.rmi.acnet.ge/pub/hha/volumes/2003/n1a13/v5n1a13.(dvi,ps,pdf)

Donald M. Davis dmd1@lehigh.edu http://www.lehigh.edu/ dmd1

Lehigh University

Bethlehem, PA 18015 